INDEXES OF HYDROLOGIC DATA FROM SELECTED COAL-MINING

AREAS IN NORTHWESTERN COLORADO

By Neville G. Gaggiani

U.S. GEOLOGICAL SURVEY

Open-File Report 88-347

Prepared in cooperation with the

U.S. BUREAU OF LAND MANAGEMENT and the

COLORADO DEPARTMENT OF NATURAL RESOURCES,

MINED LAND RECLAMATION DIVISION

Denver, Colorado 1989 
DEPARTMENT OF THE INTERIOR

MANUEL LUJAN, JR., Secretary

U.S. GEOLOGICAL SURVEY

Dallas L. Peck, Director

For additional information write to:

District Chief

U.S. Geological Survey

Box 25046, Mail Stop 415

Federal Center

Denver, CO 80225-0046
Copies of this report can be purchased from:

U.S. Geological Survey

Books and Open-File Reports Section

Federal Center

Box 25425

Denver, CO 80225-0425

[Telephone: (303) 236-7476] 
Abstract-1-

Introduction---

Purpose and scope--0.--

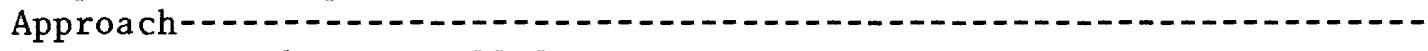

System of numbering well locations-

Acknowledgments---

Data indexes--..-

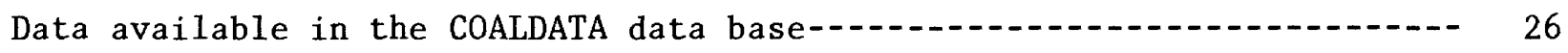

Supplemental data-

\section{PLATES}

[In back of report]

Plates 1-3. Maps showing:

1. Surface-water sites for which water-quality, discharge, or site-description data are available, northwestern Colorado

2. Ground-water sites for which water-quality, water-level, or well-construction data collected by agencies other than the U.S. Geological Survey are available, northwestern Colorado

3. Ground-water sites for which water-quality, water-level, or well-construction data collected by the U.S. Geological Survey are available, northwestern Colorado

\section{FIGURES}

Figure 1. Map showing location of study area and coal mines-1.-.

2. Diagram showing well-numbering system-- 8

\section{TABLES}

Table 1. Water-quality constituents used in the COALDATA data base for this report-... 3

2. Surface-water site descriptions and availability of data collected by agencies other than the U.S. Geological Survey-urface-water site descriptions and availability of data

3. Surface-water site descriptions and availability of data
collected by the U.S. Geological Survey-a

4. Ground-water site descriptions and availability of data collected by agencies other than the U.S. Geological Survey-- 14

5. Ground-water site descriptions and availability of data collected by the U.S. Geological Survey-16

6-8. Example printout of:

6. U.S. Geological Survey stream-discharge data---.-.-- 28

7. Ground-water levels- 29

8. U.S. Geological Survey water-quality data-- 30 


\section{CONVERSION FACTORS}

Inch-pound units in this report may be converted to metric (International System) units by using the following conversion factors:

Multiply

cubic foot per second $\left(\mathrm{ft}^{3} / \mathrm{s}\right)$

foot $(\mathrm{ft})$

mile
By

0.001233

0.3048

1.609
To obtain

cubic hectometer

meter

kilometer

Degree Celsius $\left({ }^{\circ} \mathrm{C}\right)$ may be converted to degree Fahrenheit $\left({ }^{\circ} \mathrm{F}\right)$ by using the following equation:

$$
{ }^{\circ} \mathrm{F}=9 / 5\left({ }^{\circ} \mathrm{C}\right)+32
$$

Sea level: In this report "sea level" refers to the National Geodetic Vertical Datum of 1929 (NGVD of 1929)--a geodetic datum derived from a general adjustment of the first-order level nets of both the United States and Canada, formerly called Sea Level Datum of 1929.

The following terms and abbreviations also are used in this report:

degree Celsius (deg $C$ )

microgram per liter $(\mu \mathrm{g} / \mathrm{L})$

micromhos per centimeter at 25 degrees Celsius ( $\mu$ mhos $/ \mathrm{cm}$ )

microsiemens per centimeter at 25 degrees Celsius $(\mu \mathrm{S} / \mathrm{cm})$

milliequivalent per liter (meq/L)

milligram per liter (mg/L)

milliliter per liter $(\mathrm{mL} / \mathrm{L})$

millivolt $(\mathrm{mV})$ 


\title{
INDEXES OF HYDROLOGIC DATA FROM SELECTED COAL-MINING AREAS IN NORTHWESTERN COLORADO
}

By Neville G. Gaggiani

\begin{abstract}
Currently (1988), data from hydrologic studies related to coal mining that have been done in northwestern Colorado since the early $1970^{\prime}$ s are stored in the files of private companies and government offices and in various computer systems. To compile these data for additional research, a trip to each office would have to be made to determine the availability and acceptability of the data. A data base (COALDATA) was compiled that includes stream discharge, ground-water levels, and chemical analysis of water samples that were collected by private companies and government agencies other than the U.S. Geological Survey in and near selected coal mines in northwestern Colorado. Indexes in this report 1 ist 93 surface-water sites and 95 groundwater sites where hydrologic data are available in the COALDATA data base. The indexes also list 62 surface-water sites and 480 ground-water sites in the U.S. Geological Survey data base, which is separate from the COALDATA data base and contains only data collected by the U.S. Geological Survey. The combined output of the COALDATA data base and the U.S. Geological Survey data base provides surface-water and ground-water data that include most of the study area.
\end{abstract}

\section{INTRODUCTION}

Hydrologic studies related to coal mining have been done in northwestern Colorado (fig. 1) since the early 1970's. The studies, which were done by private companies and government agencies, have included collection of data from 93. surface-water sites and 95 ground-water sites. The data include continuous discharge measurements from streamflow gaging stations, intermittent discharge measurements from miscellaneous sites, ground-water level measurements, well-construction data, some geologic data, and water-quality analyses of surface water and ground water. The water-quality constituents included in the chemical analyses are listed in table 1.

Currently (1988), hydrologic data are stored in the files of private companies and government offices, and in a variety of computers. In order to compile these data in preparation for research, a trip to each office would need to be made to determine the availability and acceptability of the data. To make these data more easily available, a centralized data base and index has been developed. This data base (COALDATA), which was compiled by the U.S. Geological Survey, in cooperation with the U.S. Bureau of Land Management and 


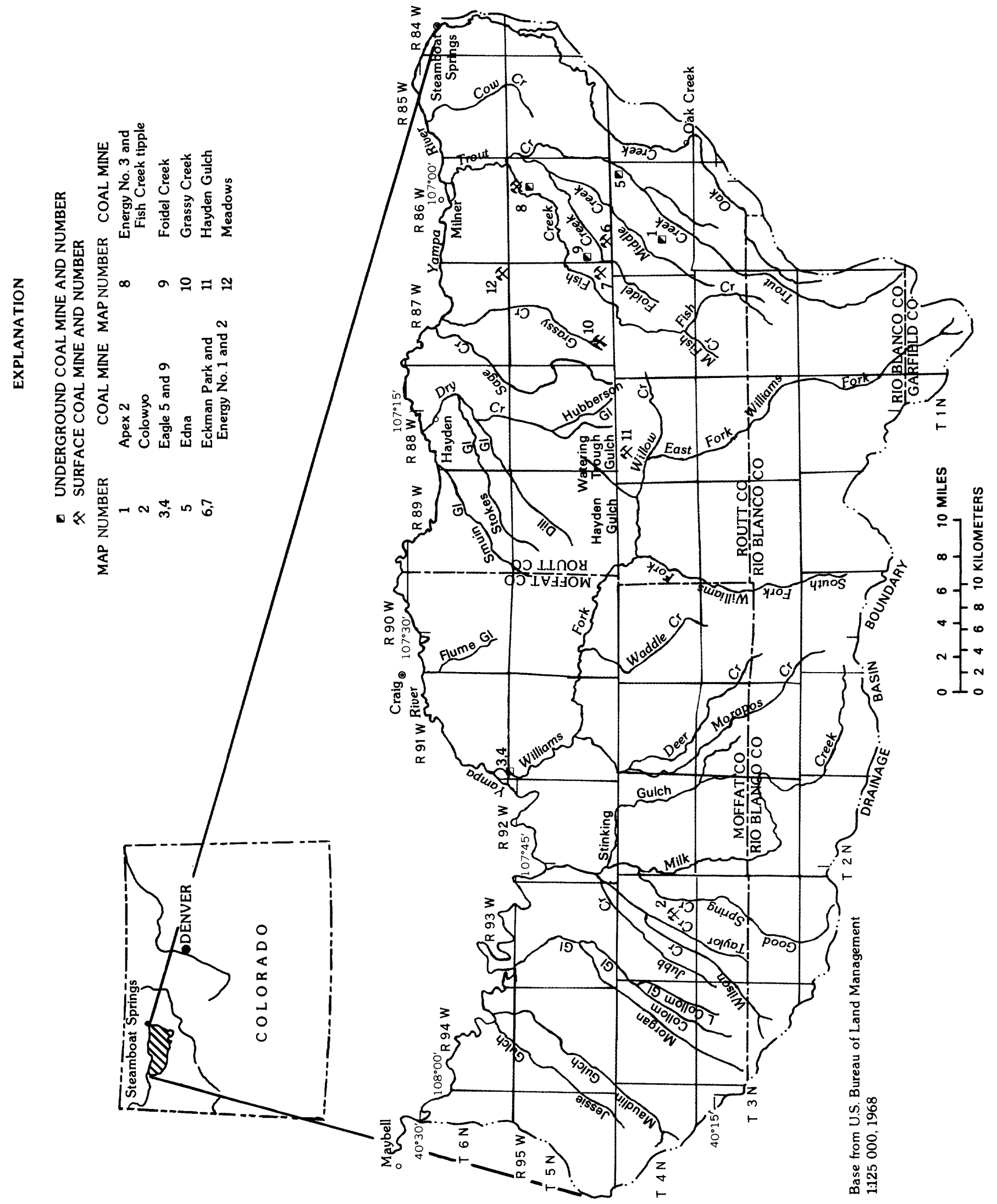

Figure 1.--Location of study area and coal mines. 
the Colorado Department of Natural Resources, Mined Land Reclamation Division, contains locations, descriptions, and hydrologic data from surface-water and ground-water sites in northwestern Colorado. In addition to the data in the COALDATA data base, the U.S. Geological Survey has hydrologic data from 62 surface-water sites and 480 ground-water sites in the study area stored in a separate U.S. Geological Survey data base.

Table 1.--Water-quality constituents used in the COALDATA data base for this report

[NWIS, National Water Information System; ${ }^{\circ} \mathrm{C}$, degrees Celsius; $\mathrm{ft}^{3} / \mathrm{s}$, cubic feet per second; $\mathrm{mV}$, millivolts; $\mu \mathrm{S} / \mathrm{cm}$, microsiemens per centimeter at 25 degrees Celsius; mg/L, milligrams per liter; meq/L, milliequivalents per liter; $\mu$ mhos $/ \mathrm{cm}$, micromhos per centimeter; $\mu \mathrm{g} / \mathrm{L}$, micrograms per liter; $\mathrm{mL} / \mathrm{L}$, milliliters per liter; ft, feet]

NWIS codes

Description of constituent

\begin{tabular}{|c|c|}
\hline 00010 & Temperature, water $\left({ }^{\circ} \mathrm{C}\right)$ \\
\hline 00060 & Discharge $\left(\mathrm{ft}^{3} / \mathrm{s}\right)$ \\
\hline 00090 & Oxidation reduction potential $(\mathrm{mV})$ \\
\hline 00095 & Specific conductance $(\mu \mathrm{S} / \mathrm{cm})$ \\
\hline 00300 & Oxygen, dissolved (mg/L) \\
\hline 00400 & $\mathrm{pH}$ (standard units) (meq/L) \\
\hline 00401 & Cations minus anions \\
\hline 00402 & Specific conductance, non-temperature corrected $(\mu \mathrm{mhos} / \mathrm{cm})$ \\
\hline 00440 & Bicarbonate ion (mg/L as $\mathrm{HCO}_{3}$ ) \\
\hline 00445 & Carbonate ion $\left(\mathrm{mg} / \mathrm{L}\right.$ as $\left.\mathrm{CO}_{3}\right)$ \\
\hline 00608 & Nitrogen, ammonia, dissolved (mg/L as $\mathrm{N}$ ) \\
\hline 00610 & Nitrogen, ammonia, total (mg/L as $\mathrm{N})$ \\
\hline 00613 & Nitrogen, nitrite, dissolved (mg/L as $\mathrm{N})$ \\
\hline 00615 & Nitrogen, nitrite, total $(\mathrm{mg} / \mathrm{L}$ as $\mathrm{N})$ \\
\hline 00618 & Nitrogen, nitrate, dissolved (mg/L as $\mathrm{N}$ ) \\
\hline 00619 & Ammonia, un-ionized (mg/L as $\mathrm{N}$ ) \\
\hline 00620 & Nitrogen, nitrate, total $(\mathrm{mg} / \mathrm{L}$ as $\mathrm{N})$ \\
\hline 00630 & Nitrogen, nitrite plus nitrate, total (mg/L as $N$ ) \\
\hline 00631 & Nitrogen, nitrite plus nitrate, dissolved (mg/L as $N$ ) \\
\hline 00650 & Phosphate, total $\left(\mathrm{mg} / \mathrm{L}\right.$ as $\left.\mathrm{PO}_{4}\right)$ \\
\hline 00653 & Phosphate, dissolved (mg/L as $\mathrm{PO}_{4}$ ) \\
\hline 00660 & Phosphate, ortho, dissolved (mg/L as $\left.\mathrm{PO}_{4}\right)$ \\
\hline 00665 & Phosphorus, total (mg/L as $\mathrm{P}$ ) \\
\hline 00745 & Sulfide, total (mg/L as $\mathrm{S})$ \\
\hline 00746 & Sulfide, dissolved (mg/L as S) \\
\hline
\end{tabular}


Table 1.--Water-quality constituents used in the COALDATA data base for this report--Continued

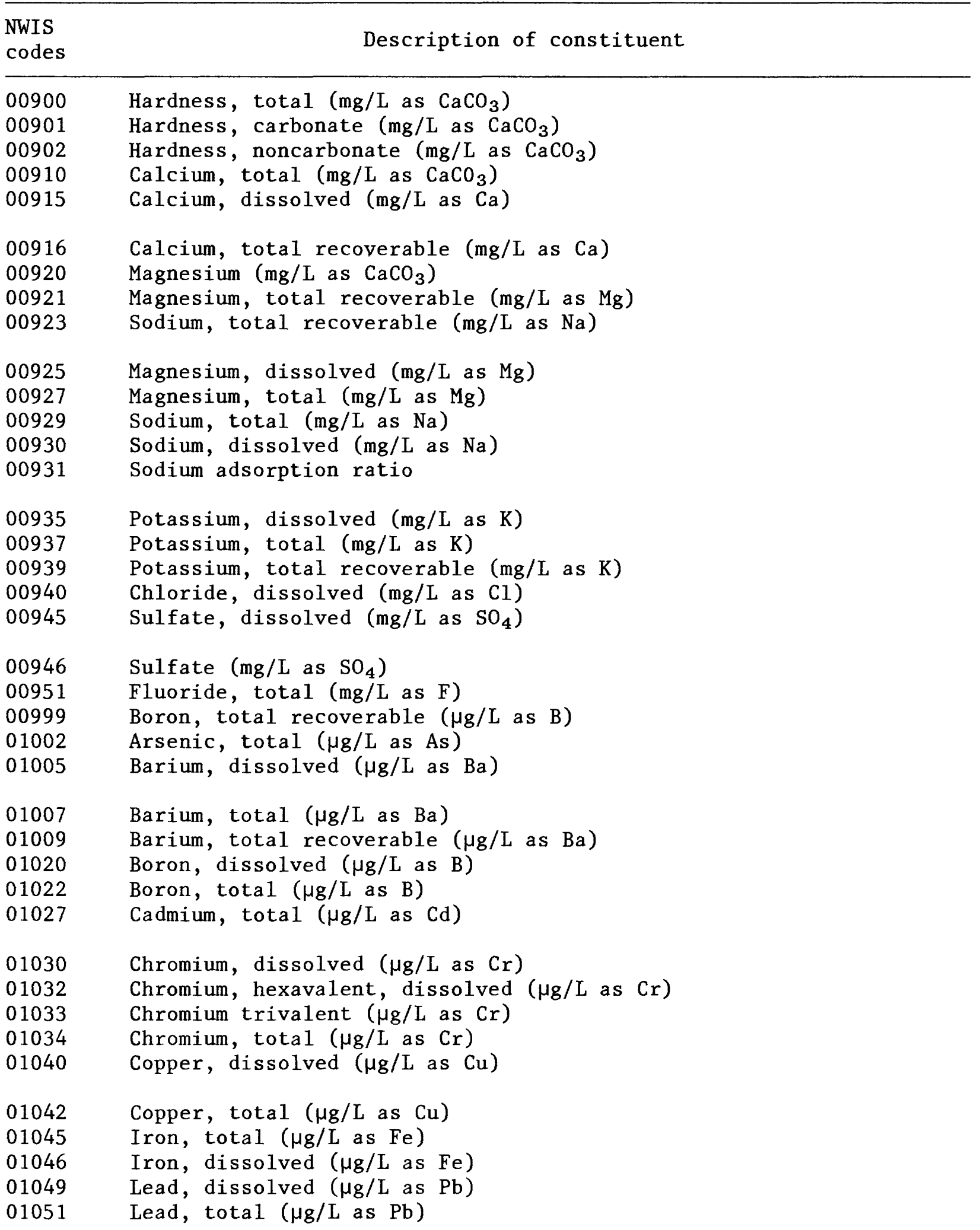


Table 1.--Water-quality constituents used in the COALDATA data base for this report--Continued

\begin{tabular}{|c|c|}
\hline $\begin{array}{l}\text { NWIS } \\
\text { codes }\end{array}$ & Description of constituent \\
\hline $\begin{array}{l}01055 \\
01056 \\
01060 \\
01062 \\
01065\end{array}$ & $\begin{array}{l}\text { Manganese, total }(\mu \mathrm{g} / \mathrm{L} \text { as Mn }) \\
\text { Manganese, dissolved }(\mu \mathrm{g} / \mathrm{L} \text { as } \mathrm{Mn}) \\
\text { Molybdenum, dissolved }(\mu \mathrm{g} / \mathrm{L} \text { as Mo }) \\
\text { Molybdenum, total }(\mu \mathrm{g} / \mathrm{L} \text { as } \mathrm{Mo}) \\
\text { Nickel, dissolved }(\mu \mathrm{g} / \mathrm{L} \text { as } \mathrm{Ni})\end{array}$ \\
\hline $\begin{array}{l}01067 \\
01074 \\
01075 \\
01077 \\
01079\end{array}$ & $\begin{array}{l}\text { Nickel, total }(\mu g / \mathrm{L} \text { as } \mathrm{Ni}) \\
\text { Nickel, total recoverable }(\mu \mathrm{g} / \mathrm{L} \text { as } \mathrm{Ni}) \\
\text { Silver, dissolved }(\mu \mathrm{g} / \mathrm{L} \text { as } \mathrm{Ag}) \\
\text { Silver, total ( } \mu \mathrm{g} / \mathrm{L} \text { as } \mathrm{Ag}) \\
\text { Silver, total recoverable }(\mu \mathrm{g} / \mathrm{L} \text { as } \mathrm{Ag})\end{array}$ \\
\hline $\begin{array}{l}01090 \\
01092 \\
01094 \\
01105 \\
01106\end{array}$ & $\begin{array}{l}\text { Zinc, dissolved }(\mu \mathrm{g} / \mathrm{L} \text { as } \mathrm{Zn}) \\
\text { Zinc, total }(\mu \mathrm{g} / \mathrm{L} \text { as } \mathrm{Zn}) \\
\text { Zinc, total recoverable }(\mu \mathrm{g} / \mathrm{L} \text { as } \mathrm{Zn}) \\
\text { Aluminum, total ( } \mu \mathrm{g} / \mathrm{L} \text { as } \mathrm{Al}) \\
\text { Aluminum, dissolved }(\mu \mathrm{g} / \mathrm{L} \text { as } \mathrm{Al})\end{array}$ \\
\hline $\begin{array}{l}01114 \\
01118 \\
01123 \\
01129 \\
01145\end{array}$ & $\begin{array}{l}\text { Lead, total recoverable }(\mu \mathrm{g} / \mathrm{L} \text { as } \mathrm{Pb}) \\
\text { Chromium, total recoverable }(\mu \mathrm{g} / \mathrm{L} \text { as } \mathrm{Cr}) \\
\text { Manganese total recoverable }(\mu \mathrm{g} / \mathrm{L} \text { as } \mathrm{Mn}) \\
\text { Molybdenum total recoverable }(\mu \mathrm{g} / \mathrm{L} \text { as } \mathrm{Mb}) \\
\text { Selenium, dissolved }(\mu \mathrm{g} / \mathrm{L} \text { as } \mathrm{Se})\end{array}$ \\
\hline $\begin{array}{l}01147 \\
01355 \\
70301 \\
70348 \\
70507\end{array}$ & $\begin{array}{l}\text { Selenium, total ( } \mu \mathrm{g} / \mathrm{L} \text { as } \mathrm{Se}) \\
\text { Ice cover, floating or solid (severity) } \\
\text { Solids, sum of constituents, dissolved (mg/L) } \\
\text { Settleable solids (mL/L) } \\
\text { Phosphorus, orthophosphate, total (mg/L as P) }\end{array}$ \\
\hline $\begin{array}{l}71825 \\
71900 \\
72019 \\
80154 \\
90410\end{array}$ & $\begin{array}{l}\text { Acidity, total (mg/L as } \mathrm{H}) \\
\text { Mercury, total recoverable }(\mu \mathrm{g} / \mathrm{L} \text { as } \mathrm{Hg}) \\
\text { Depth below land surface (water level) (ft) } \\
\text { Sediment, suspended concentration (mg/L) } \\
\text { Alkalinity }\left(\mathrm{mg} / \mathrm{L} \text { as } \mathrm{CaCO}_{3}\right)\end{array}$ \\
\hline
\end{tabular}




\section{Purpose and Scope}

This report describes the type of data that are available in COALDATA and gives examples of the types of information retrievals that are available from the system. Because the data base is large, actual data stored in the data base are not included in this report. Some descriptive information about the sites to aid in site selection are included in tables 2 through 5.

The data have been collected from private sources such as coal companies in northwestern Colorado and government sources such as the Colorado Department of Natural Resources, Mined Land Reclamation Division. Surfacewater data include site location, type of gage, ownership, and surface-water discharge values. Ground-water data include well location, well depth, ownership (name of mine), depth of water-producing zone, and name of aquifer. Water-quality data include chemical analyses of selected constituents. Onsite water-quality measurements such as temperature, specific conductance, $\mathrm{pH}$, and dissolved oxygen also are included, if available.

Hydrologic data stored in COALDATA have not been checked by the U.S. Geological Survey; also data collection and analysis procedures have not been verified to ensure that they meet standard U.S. Geological Survey methods and techniques. Therefore, proper data accuracy determination and appropriate data application are left to the user's discretion.

\section{Approach}

Several methods were used to transfer data to the COALDATA data base. One method used was to manually enter data from reports and paper records. A second method of data transfer was to transfer the hydrologic data to magnetic tape from the host computer and then read the tape into the U.S. Geological Survey computer.

The COALDATA data base was developed using the U.S. Geological Survey National Water Information system (NWIS). This system processes and stores data in the COALDATA data base using two programs: the ground-water site inventory program (GWSI), which processes data for ground-water levels and information related to the drilling and operation of the site; and the quality of water program (QWDATA), which processes data for onsite measurements, chemical laboratory analyses of surface and ground water, and data for instantaneous surface-water discharge measurements. Site description information is stored so that it is available to both programs. 


\section{System of Numbering We11 Locations}

The well locations (local well number) in tables 4 and 5 are based on the U.S. Bureau of Land Management system of land subdivision, and indicate the location of the well by quadrant, township, range, section, and position within the section. A graphic illustration of this method of well location is shown in figure 2. The first letter "S" preceeding the location number means that the well is located in the area governed by the sixth principal meridian. The second letter indicates the quadrant in which the well is located. Four quadrants are formed by the intersection of the base line and the principal meridian--A indicates the northeast quadrant, B the northwest, $C$ the southwest, and $D$ the southeast. The first numeral indicates the township, the second the range, and the third the section in which the well is located. The letters following the section number locate the well within the section. The first letter denotes the quarter section, the second the quarter-quarter section. The letters are assigned within the section in a counter-clockwise direction, beginning with (A) in the northeast quarter. Letters are assigned within each quarter section and within each quarter-quarter section in the same manner. Where two or more locations are within the smallest subdivision, consecutive numbers beginning with 1 are added in the order in which the wells were inventoried. For example, SC6-47-16AAA indicates a well in the northeast quarter of the northeast quarter of the northeast quarter of sec. 16, T. 6 S., $\mathrm{R} .47 \mathrm{~W}$. The " $\mathrm{S}$ " refers to the sixth principal meridian. The "C" indicates the township is south of the base line and that the range is west of the principal meridian.

\section{Acknowledgments}

Bob Liddle, Candy Thompson, and Jim Pendelton of the Colorado Mined Land Reclamation Division helped compile the data, and Gary McIntosh of the U.S. Office of Surface Mining helped with writing computer programs. Their assistance is gratefully appreciated.

\section{DATA INDEXES}

Indexes of surface-water and ground-water sites in and near selected coal mines in northwestern Colorado are listed in tables 2 through 5 . The sites that are in the COALDATA data base, which contains only data that were collected by agencies other than the U.S. Geological Survey are 1isted in tables 2 and 4 . The sites that are in the main U.S Geological Survey data base, which contains only data collected by the U.S Geological Survey are listed in tables 3 and 5. Data retrievals combining the U.S. Geological Survey files and COALDATA files can be combined into one output, since these two data bases use the same computer system. Locations of these sites are indicated in maps on plates 1 through 3 . 


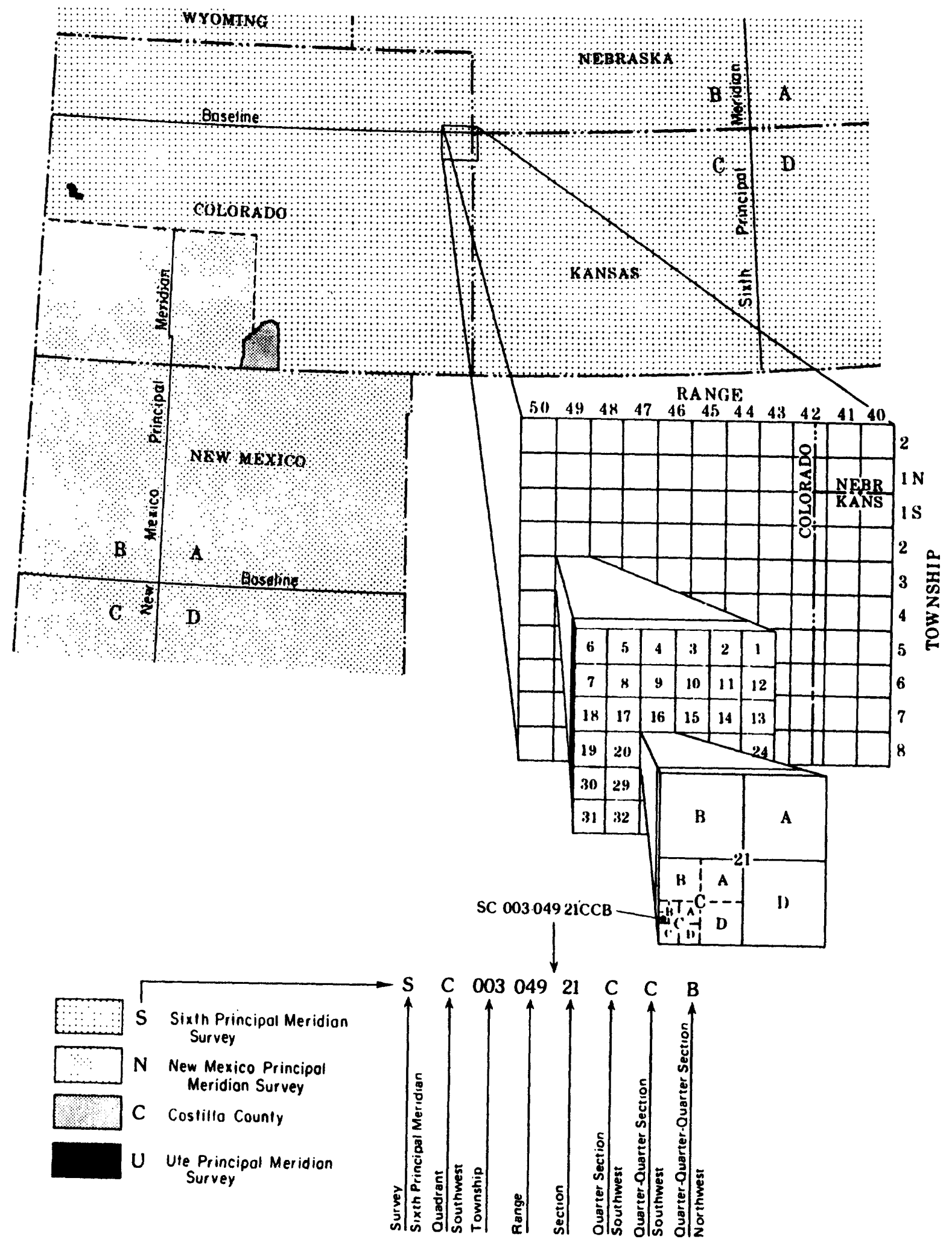

Figure 2.--Well-numbering system. 


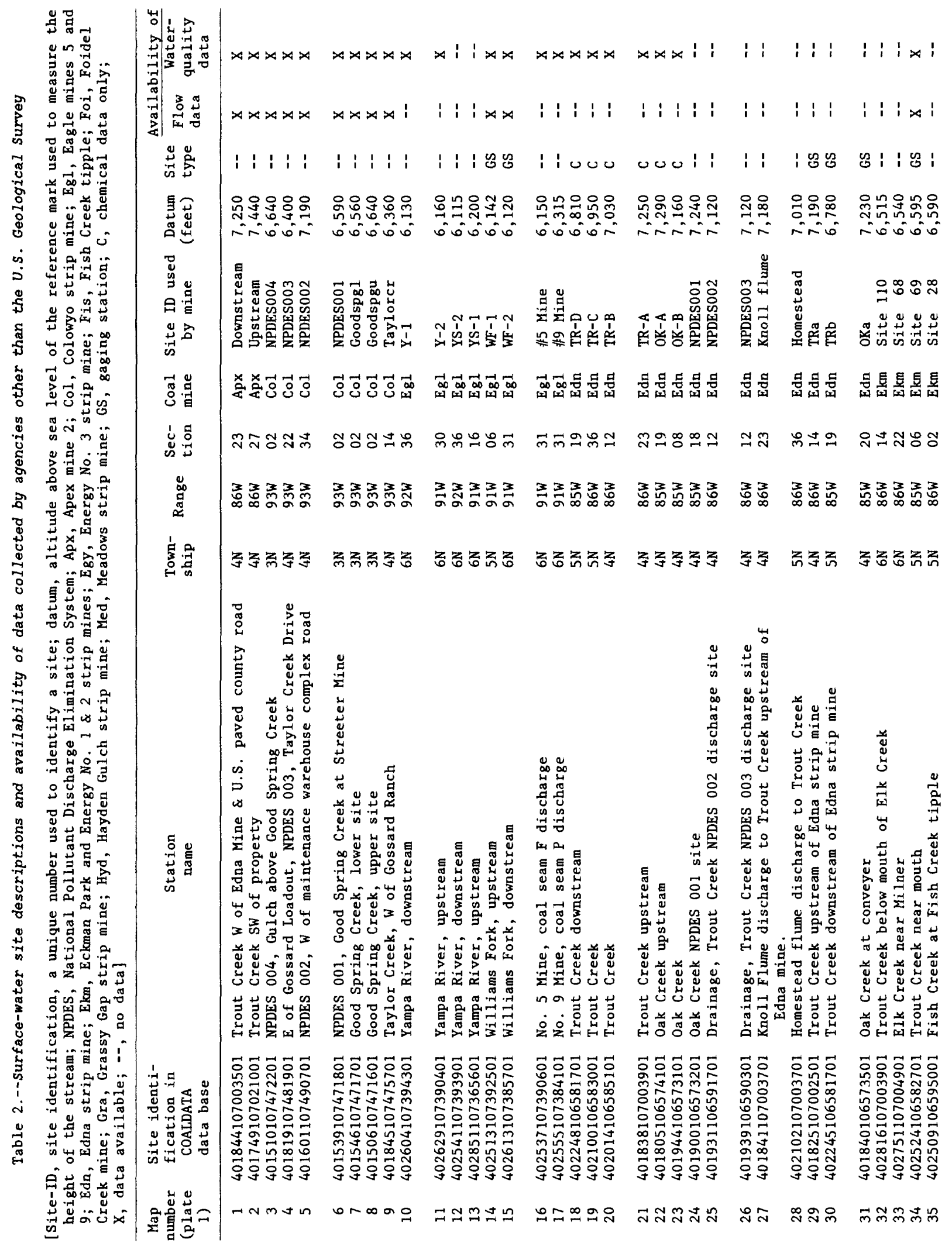




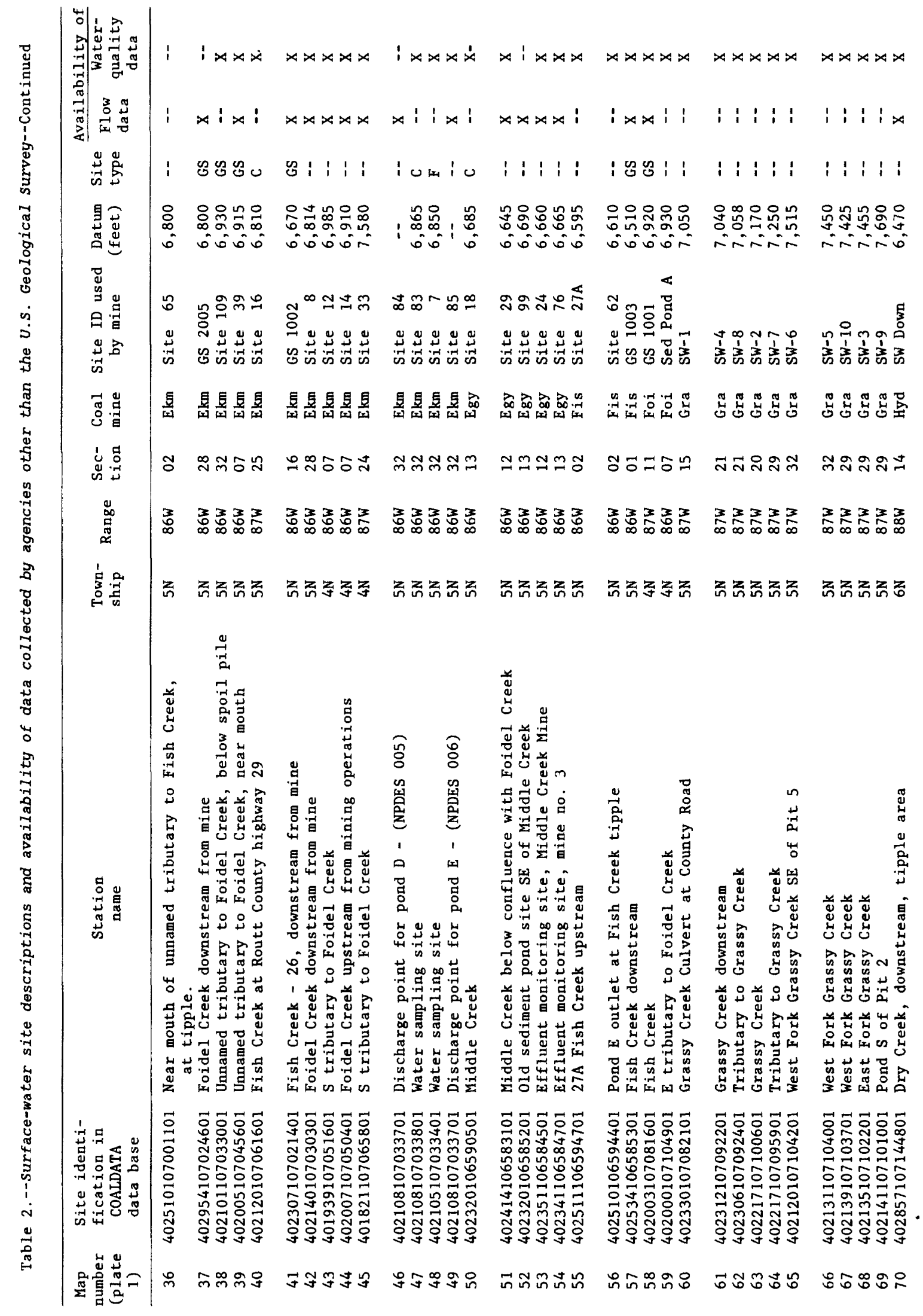




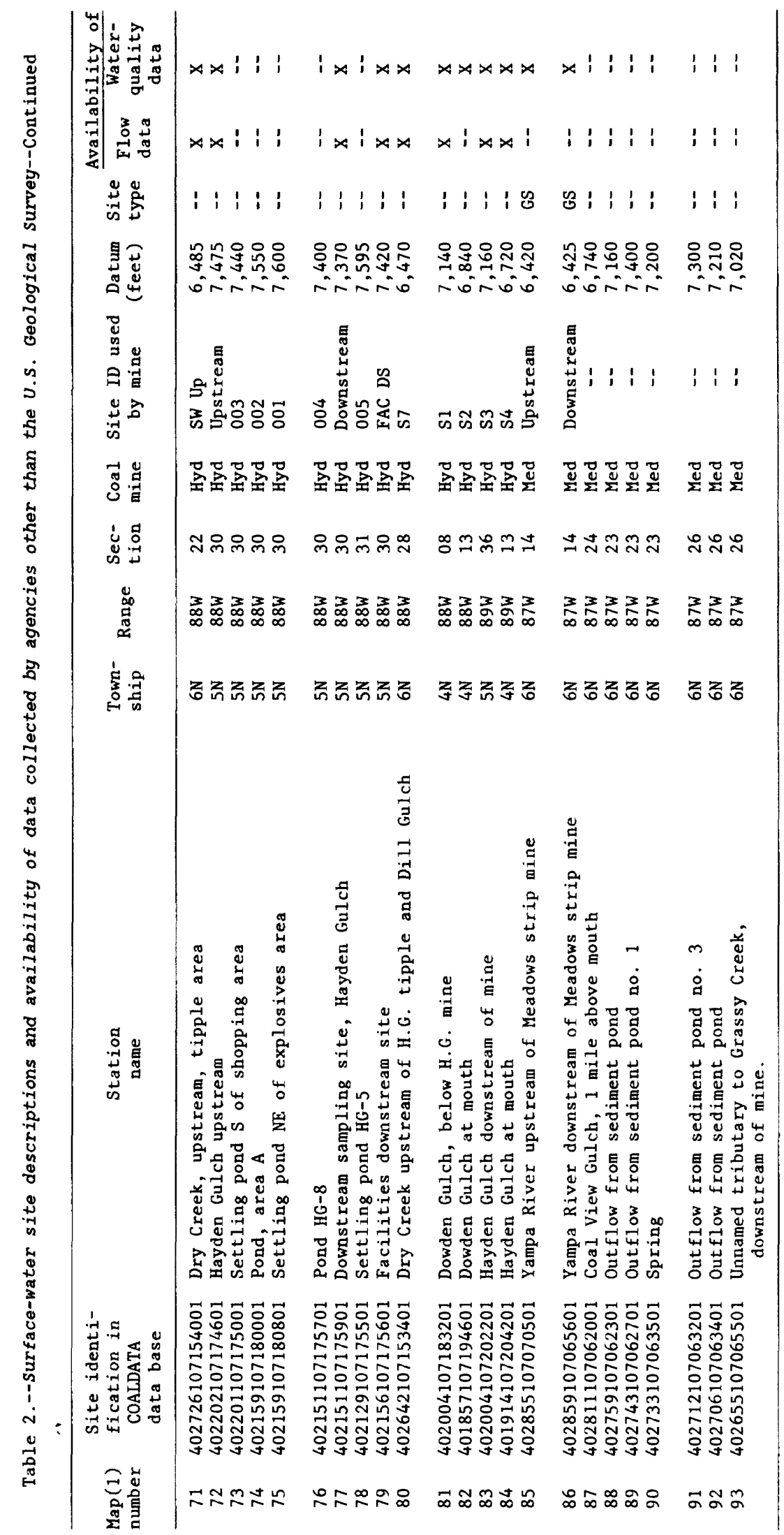




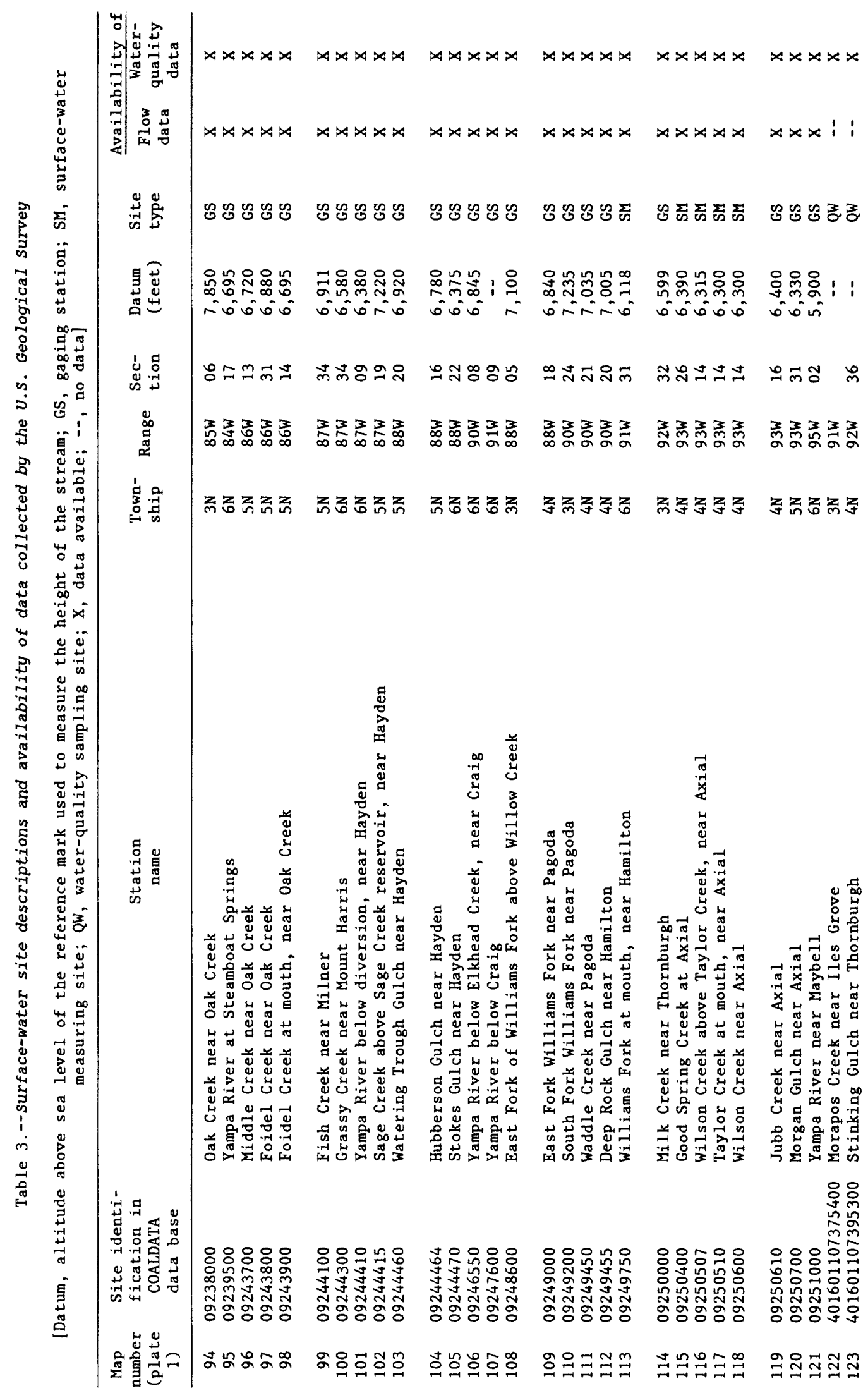




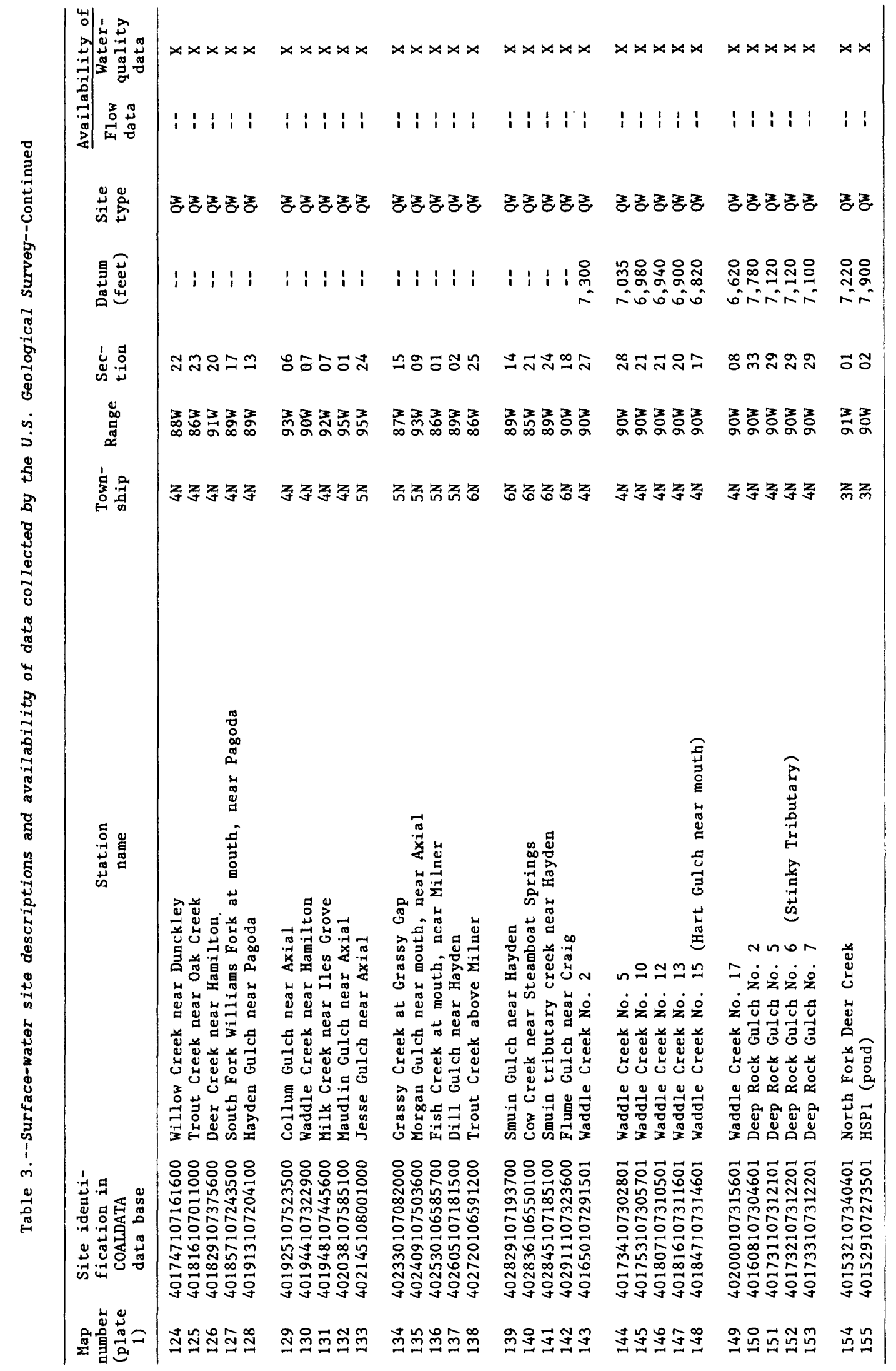


Table 4.--Ground-water site descriptions and availability of data collected by agencies other than the U.S. Geological Survey

[Site-ID, site identification, a unique number used to identify a site; Datum, altitude above sea level of the reference mark used to measure the height of the stream; Apx, Apex mine number 2; Col, Colowyo strip mine; Edn, Edna strip mine; Egl, Eagle mines 5 and 9 ; Egy, Energy No. 3 strip mine; Ekm, Eckman Park and Energy No. 1 \& 2 strip mines; Fis, Fish Creek tipple; Foi, Foidel Creek mine; Gra, Grassy Gap strip mine; Hyd, Hayden Gulch strip mine; Med, Meadows strip mine; $X$, data available; --, no data]

\begin{tabular}{|c|c|c|c|c|c|c|c|c|c|c|}
\hline \multirow{2}{*}{$\begin{array}{l}\text { Map } \\
\text { number } \\
\text { (plate } \\
2 \text { ) }\end{array}$} & \multirow{2}{*}{$\begin{array}{l}\text { Site identi- } \\
\text { fication in } \\
\text { COALDATA } \\
\text { data base }\end{array}$} & \multirow[b]{2}{*}{$\begin{array}{c}\text { Local well } \\
\text { number } \\
\text { (see fig. 2) }\end{array}$} & \multirow[b]{2}{*}{ Latitude } & \multirow[b]{2}{*}{ Longitude } & \multirow[b]{2}{*}{ Mine } & \multirow[b]{2}{*}{$\begin{array}{l}\text { Site-ID } \\
\text { used by } \\
\text { mine }\end{array}$} & \multirow[b]{2}{*}{$\begin{array}{l}\text { Datum } \\
\text { (feet) }\end{array}$} & \multicolumn{3}{|c|}{ Availability of } \\
\hline & & & & & & & & $\begin{array}{l}\text { Water- } \\
\text { level } \\
\text { data }\end{array}$ & $\begin{array}{l}\text { Water- } \\
\text { quality } \\
\text { data }\end{array}$ & $\begin{array}{l}\text { Well-con- } \\
\text { struction } \\
\text { data }\end{array}$ \\
\hline 1 & 401902107002001 & SB00408614DBB & $40^{\circ} 19^{\prime} 02^{\prime \prime}$ & $107^{\circ} 00^{\prime} 20^{\prime \prime}$ & Apx & SEC1 4NWSE & 7,180 & -- & $\mathbf{X}$ & $\mathrm{X}$ \\
\hline 2 & 401753107020101 & SB00408622CCC & $40^{\circ} 27^{\prime} 53^{\prime \prime}$ & $107^{\circ} 02^{\prime} 17^{\prime \prime}$ & Apx & SEC22SWSW & 7,420 & -- & $\mathrm{X}$ & $\mathrm{X}$ \\
\hline 3 & 401541107472201 & SB00309302ACB & $40^{\circ} 15^{\prime} 41^{\prime \prime}$ & $107^{\circ} 47^{\prime} 22^{\prime \prime}$ & Col & $6600 \mathrm{~N}$ & 6,580 & $\mathbf{X}$ & $\mathbf{X}$ & -- \\
\hline 4 & 401533107472101 & SB00309302ACD & $40^{\circ} 15^{\prime} 33^{\prime \prime}$ & $107^{\circ} 47^{\prime} 21^{\prime \prime}$ & Col & $6600 \mathrm{~S}$ & 6,590 & $\mathrm{X}$ & $X$ & -- \\
\hline 5 & 401539107473401 & SB00309302BDA & $40^{\circ} 15^{\prime} 33^{\prime \prime}$ & $107^{\circ} 47^{\prime} 21^{\prime \prime}$ & Col & $6900 \mathrm{~N}$ & 6,700 & $x$ & $\mathrm{X}$ & -- \\
\hline 6 & 401535107473301 & SB00309302BDD & $40^{\circ} 15^{\prime} 35^{\prime \prime}$ & $107^{\circ} 47^{\prime} 33^{\prime \prime}$ & Col & $6900 \mathrm{~S}$ & 6,760 & $\mathrm{X}$ & $\mathrm{X}$ & -- \\
\hline 7 & 401832107482701 & SB00409315DDC & $40^{\circ} 18^{\prime} 32^{\prime \prime}$ & $107^{\circ} 48^{\prime} 27^{\prime \prime}$ & Col & GOSSARD WL & 6,370 & $\mathrm{X}$ & $\mathbf{X}$ & -- \\
\hline 8 & 402204106582701 & SB00508519BAC & $40^{\circ} 22^{\prime} 04^{\prime \prime}$ & $106^{\circ} 58^{\prime} 27^{\prime \prime}$ & Edn & $T R-4$ & 6,900 & -- & $X$ & $\mathrm{X}$ \\
\hline 9 & 402103106583001 & SB00508636AAD & $40^{\circ} 21^{\prime} 03^{\prime \prime}$ & $106^{\circ} 58^{\prime} 30^{\prime \prime}$ & Edn & TR-3 & 6,920 & -- & $x$ & $\mathrm{X}$ \\
\hline 10 & 402017106585101 & SB00408612AAC & $40^{\circ} 20^{\prime} 17^{\prime \prime}$ & $106^{\circ} 58^{\prime} 51^{\prime \prime}$ & Edn & $T R-2$ & 7,050 & -- & $\mathrm{X}$ & $\mathrm{X}$ \\
\hline 11 & 401909107000201 & SB00408614 & $40^{\circ} 19^{\prime} 09^{\prime \prime}$ & $107^{\circ} 00^{\prime} 02^{\prime \prime}$ & Edn & $T R-1.5$ & 7,160 & -- & $\mathrm{X}$ & $\mathrm{X}$ \\
\hline 12 & 401904107001101 & SB00408614DAB & $40^{\circ} 19^{\prime} 04^{\prime \prime}$ & $107^{\circ} 00^{\prime} 11^{\prime \prime}$ & Edn & TR-1 & 7,160 & -- & $\mathrm{X}$ & $\mathrm{X}$ \\
\hline 13 & 401917106573101 & SB00408517BCC & $40^{\circ} 19^{\prime} 17^{\prime \prime}$ & $106^{\circ} 57^{\prime} 31^{\prime \prime}$ & Edn & OK -2 & 7,210 & -- & $\mathrm{X}$ & $\mathrm{X}$ \\
\hline 14 & 401802106574201 & SB004085 19DDA & $40^{\circ} 18^{\prime} 02^{\prime \prime}$ & $106^{\circ} 57^{\prime} 42^{\prime \prime}$ & Edn & $\mathrm{OK}-1$ & 7,280 & -- & $\mathrm{X}$ & $\mathrm{X}$ \\
\hline 15 & 402540107391801 & SB00609131CCC & $40^{\circ} 25^{\prime} 40^{\prime \prime}$ & $107^{\circ} 39^{\prime} 18^{\prime \prime}$ & Eg1 & \#5MINEWELL & 6,190 & -- & $\mathrm{X}$ & $\mathrm{X}$ \\
\hline 16 & 402540107391802 & SB00509106CA & $40^{\circ} 25^{\prime} 40^{\prime \prime}$ & $107^{\circ} 39^{\prime} 18^{\prime \prime}$ & Egl & \#5A WELL & 6,190 & -- & $\mathbf{X}$ & $\mathrm{X}$ \\
\hline 17 & 402525107381301 & SB00509106AAD & $40^{\circ} 25^{\prime} 25^{\prime \prime}$ & $107^{\circ} 38^{\prime} 13^{\prime \prime}$ & $\mathrm{Eg} 1$ & OKIE PLAZA & 6,552 & -- & $\mathrm{X}$ & $\mathrm{X}$ \\
\hline 18 & 402645107373601 & SB00609129DBC & $40^{\circ} 25^{\prime} 45^{\prime \prime}$ & $107^{\circ} 37^{\prime} 36^{\prime \prime}$ & Egl & TR -4 & 6,304 & -- & $\mathrm{X}$ & $\mathrm{X}$ \\
\hline 19 & 402628107385801 & SB00609131BAB & $40^{\circ} 26^{\prime} 28^{\prime \prime}$ & $107^{\circ} 38^{\prime} 58^{\prime \prime}$ & $\mathrm{Egl}$ & TR-7A & 6,220 & -- & $\mathrm{X}$ & $\mathrm{X}$ \\
\hline 20 & 402546107381401 & SB00609131DDA & $40^{\circ} 25^{\prime} 46^{\prime \prime}$ & $107^{\circ} 38^{\prime} 14^{\prime \prime}$ & Egl & $81-01$ & 6,413 & -- & $\mathrm{X}$ & $\mathbf{x}$ \\
\hline 21 & 402614107385101 & SB00609131BDA & $40^{\circ} 26^{\prime} 14^{\prime \prime}$ & $107^{\circ} 38^{\prime} 51^{\prime \prime}$ & $E_{\mathbf{g} 1}$ & $83-01$ & 6,173 & -- & $\mathrm{X}$ & $\mathbf{X}$ \\
\hline 22 & 402534107371201 & SB00509105AAA & $40^{\circ} 25^{\prime} 34^{\prime \prime}$ & $107^{\circ} 37^{\prime} 12^{\prime \prime}$ & Egl & $83-02$ & 6,683 & -- & $\mathrm{X}$ & $\mathrm{x}$ \\
\hline 23 & 402740107382701 & SB00609119DAC & $40^{\circ} 27^{\prime} 40^{\prime \prime}$ & $107^{\circ} 38^{\prime} 27^{\prime \prime}$ & Egl & $83-03$ & 6,131 & -- & $\mathrm{X}$ & $\mathrm{x}$ \\
\hline 24 & 402615107385401 & SB00609131BAD & $40^{\circ} 26^{\prime} 15^{\prime \prime}$ & $107^{\circ} 38^{\prime} 54^{\prime \prime}$ & Egl & 259 & 6,128 & -- & $\mathrm{X}$ & $\mathrm{x}$ \\
\hline 25 & 402644107373601 & SB00609129DCB & $40^{\circ} 26^{\prime} 44^{\prime \prime}$ & $107^{\circ} 37^{\prime} 36^{\prime \prime}$ & Egl & $84-01$ & 6,307 & -- & $\mathrm{X}$ & $\mathrm{X}$ \\
\hline 26 & 402608107380401 & SB00609132BCA & $40^{\circ} 26^{\circ} 08^{\prime \prime}$ & $107^{\circ} 38^{\prime} 04^{\prime \prime}$ & Eg1 & \#1MMINEWELL & 6,383 & -- & $\mathrm{X}$ & -- \\
\hline 27 & 402706107372901 & SB00609129ACB & $40^{\circ} 27^{\prime} 06^{\prime \prime}$ & $107^{\circ} 37^{\prime} 39^{\prime \prime}$ & Egl & $81-03 A$ & 6,227 & -- & -- & -- \\
\hline 28 & 402725107391001 & SB00609119CDD & $40^{\circ} 27^{\prime} 25^{\prime \prime}$ & $107^{\circ} 39^{\prime} 10^{\prime \prime}$ & Egl & $81-04 A$ & 6,378 & -- & $\mathrm{X}$ & $\mathbf{X}$ \\
\hline 29 & 402818107375201 & SB00609117CDC & $40^{\circ} 28^{\prime} 18^{\prime \prime}$ & $107^{\circ} 37^{\prime} 52^{\prime \prime}$ & Egl & $81-19$ & 6,138 & -- & $\mathrm{X}$ & $\mathrm{X}$ \\
\hline 30 & 402631107374401 & SB00609129CDD & $40^{\circ} 26^{\prime} 31^{\prime \prime}$ & $107^{\circ} 37^{\prime} 44^{\prime \prime}$ & Eg1 & NORTHSPRNG & 6,310 & -- & $\mathrm{X}$ & Spring \\
\hline 31 & 402636107401601 & SB00609125BAC & $40^{\circ} 26^{\prime} 36^{\prime \prime}$ & $107^{\circ} 40^{\prime} 16^{\prime \prime}$ & Eg1 & HAXTONSPRG & 6,600 & -- & $\mathrm{X}$ & Spring \\
\hline 32 & 402613107385101 & SB00609131BDA & $40^{\circ} 26^{\prime} 13^{\prime \prime}$ & $107^{\circ} 38^{\circ} 51^{\prime \prime}$ & $\mathbf{E}_{\mathbf{g} 1}$ & 非1STRIPPIT & 6,170 & -- & $\mathrm{X}$ & Spring \\
\hline 33 & 402746107381901 & SB00609119DAA & $40^{\circ} 27^{\prime} 46^{\prime \prime}$ & $107^{\circ} 38^{\prime} 19^{\prime \prime}$ & Egl & YAW-1 & 6,130 & -- & $\mathrm{X}$ & $X$ \\
\hline 34 & 402732107382701 & SB00609119DDB & $40^{\circ} 27^{\prime} 32^{\prime \prime}$ & $107^{\circ} 38^{\prime} 27^{\prime \prime}$ & Egl & YAW-2 & 6,130 & -- & $\mathrm{X}$ & $\mathrm{x}$ \\
\hline 35 & 402706107380101 & SB00609129BCA & $40^{\circ} 27^{\prime} 06^{\prime \prime}$ & $107^{\circ} 38^{\prime} 01^{\prime \prime}$ & Egl & YAW-3 & 6,130 & -- & $\mathrm{X}$ & $\mathrm{x}$ \\
\hline 36 & 402718107383801 & SB00609130ABA1 & $40^{\circ} 27^{\prime} 18^{\prime \prime}$ & $107^{\circ} 38^{\prime} 38^{\prime \prime}$ & Egl & YAW-5 & 6,130 & -- & $\mathbf{X}$ & $\mathrm{X}$ \\
\hline 37 & 402718107383601 & SB00609130ABA2 & $40^{\circ} 27^{\prime} 18^{\prime \prime}$ & $107^{\circ} 38^{\prime} 36^{\prime \prime}$ & Egl & YAW-6 & 6,130 & -- & $\mathrm{x}$ & $\mathrm{x}$ \\
\hline 38 & 402550107385901 & SB0060913ICAC & $40^{\circ} 25^{\prime} 50^{\prime \prime}$ & $107^{\circ} 38^{\prime} 59^{\prime \prime}$ & Egl & AVF-3 & 6,135 & -- & $\mathrm{x}$ & $\mathrm{X}$ \\
\hline 39 & 402601107385401 & SB00609131BDD & $40^{\circ} 26^{\circ} 01^{\prime \prime}$ & $107^{\circ} 39^{\prime} 18^{\prime \prime}$ & Egl & AVF -5 & 6,135 & -- & $\mathrm{X}$ & $\mathrm{X}$ \\
\hline 40 & 402535107391801 & SB00509106BBB & $40^{\circ} 25^{\prime} 35^{\prime \prime}$ & $107^{\circ} 39^{\prime} 18^{\prime \prime}$ & Egl & AVF -6 & 6,140 & -- & $\mathrm{x}$ & -- \\
\hline 41 & 402327106585601 & SB00508613 & $40^{\circ} 23^{\prime} 27^{\prime t}$ & $106^{\circ} 58^{\prime} 56^{\prime \prime}$ & Egy & $008-A V-1$ & 6,679 & -- & $\mathbf{X}$ & $\mathrm{X}$ \\
\hline 42 & 402345106585301 & SB00508613ABC & $40^{\circ} 23^{\prime} 45^{\prime \prime}$ & $106^{\circ} 58^{\prime} 53^{\prime \prime}$ & Egy & $008-A V-2$ & 6,676 & -- & $\mathbf{X}$ & $\mathrm{X}$ \\
\hline 43 & 402344106585101 & SB00508613 & $40^{\circ} 23^{\prime} 44^{\prime \prime}$ & $106^{\circ} 58^{\prime} 51^{\prime \prime}$ & Egy & $008-A V-3$ & 6,676 & -- & $\mathrm{X}$ & $\mathrm{X}$ \\
\hline 44 & 402343106585001 & SB00508613 & $40^{\circ} 23^{\prime} 43^{\prime \prime}$ & $106^{\circ} 58^{\prime} 50^{\prime \prime}$ & Egy & $008-A V-4$ & 6,676 & -- & $\mathrm{x}$ & $\mathrm{X}$ \\
\hline 45 & 402414106583001 & SB00508612 & $40^{\circ} 24^{\prime} 14^{\prime \prime}$ & $106^{\circ} 58^{\prime} 30^{\prime \prime}$ & Egy & $008-A X-1$ & 6,655 & - & $\mathrm{x}$ & $\mathrm{X}$ \\
\hline 46 & 402344106585901 & SB00508613 & $40^{\circ} 23^{\prime} 44^{\prime \prime}$ & $106^{\circ} 58^{\prime} 59^{\prime \prime}$ & Egy & 008-FM-6 & 6,673 & -- & $\mathbf{X}$ & $\mathrm{X}$ \\
\hline 47 & 402339106585601 & SB00508613ABD & $40^{\circ} 23^{\prime} 39^{\prime \prime}$ & $106^{\circ} 58^{\prime} 56^{\prime \prime}$ & Egy & $008-77-35$ & 6,673 & -- & $\mathrm{x}$ & $\mathrm{X}$ \\
\hline 48 & 402313106585501 & SBO0508613DBC & $40^{\circ} 23^{\prime} 13^{\prime \prime}$ & $106^{\circ} 58^{\prime} 55^{\prime \prime}$ & Egy & $008-77-42$ & 6,741 & -- & $\mathrm{X}$ & $\mathrm{X}$ \\
\hline 49 & 402313106584701 & SB00508613DCA & $40^{\circ} 23^{\prime} 13^{\prime \prime}$ & $106^{\circ} 58^{\prime} 47^{\prime \prime}$ & Egy & $008-77-43$ & 6,714 & -- & $\mathrm{X}$ & $\mathrm{X}$ \\
\hline 50 & 402320106584501 & SB00508613DBA & $40^{\circ} 23^{\prime} 20^{\prime \prime}$ & $106^{\circ} 58^{\prime} 45^{\prime \prime}$ & Egy & $008-77-58$ & 6,720 & -- & $\mathrm{X}$ & $\mathrm{X}$ \\
\hline
\end{tabular}


Table 4,--Ground-water site descriptions and availability of data collected by agencies other than the U.S. Geological Survey--Continued

\begin{tabular}{|c|c|c|c|c|c|c|c|c|c|c|}
\hline \multirow{2}{*}{$\begin{array}{l}\text { Map } \\
\text { number } \\
\text { (plate } \\
2 \text { ) }\end{array}$} & \multirow{2}{*}{$\begin{array}{l}\text { Site identi- } \\
\text { fication in } \\
\text { COALDATA } \\
\text { data base }\end{array}$} & \multirow[b]{2}{*}{$\begin{array}{c}\text { Local well } \\
\text { number } \\
\text { (see fig. 2) }\end{array}$} & \multirow[b]{2}{*}{ Latitude } & \multirow[b]{2}{*}{ Longitude } & \multirow[b]{2}{*}{ Mine } & \multirow[b]{2}{*}{$\begin{array}{l}\text { Site-ID } \\
\text { used by } \\
\text { mine }\end{array}$} & \multirow[b]{2}{*}{$\begin{array}{l}\text { Datum } \\
\text { (feet) }\end{array}$} & \multicolumn{3}{|c|}{ Availability of } \\
\hline & & & & & & & & $\begin{array}{l}\text { Water- } \\
\text { level } \\
\text { data }\end{array}$ & $\begin{array}{l}\text { Water- } \\
\text { quality } \\
\text { data }\end{array}$ & $\begin{array}{l}\text { Well-con- } \\
\text { struction } \\
\text { data }\end{array}$ \\
\hline 51 & 402344106585801 & SB00508613ABC1 & $40^{\circ} 23^{\prime} 44^{\prime \prime}$ & $106^{\circ} 58^{\prime} 58^{\prime \prime}$ & Egy & $008-79-6$ & 6,676 & -- & $\mathrm{X}$ & $\mathrm{X}$ \\
\hline 52 & 402346106585901 & SB00508613ABC2 & $40^{\circ} 23^{\prime} 46^{\prime \prime}$ & $106^{\circ} 58^{\prime} 59^{\prime \prime}$ & Egy & $008-79-7$ & 6,696 & -- & $\mathrm{X}$ & $\mathrm{X}$ \\
\hline 53 & 402341106584201 & SB00508613 & $40^{\circ} 23^{\prime} 41^{\prime \prime}$ & $106^{\circ} 58^{\prime} 42^{\prime \prime}$ & Egy & $008-A Y-2$ & 6,680 & -- & $\mathrm{X}$ & -- \\
\hline 54 & 402509107003001 & SB00508602 & $40^{\circ} 25^{\prime} 09^{\prime \prime}$ & $107^{\circ} 00^{\prime} 30^{\prime \prime}$ & Egy & $008-\mathrm{AU}-1$ & 6,616 & -- & $\mathrm{X}$ & $\mathrm{x}$ \\
\hline 56 & 402300107033301 & SB00508616DCA & $40^{\circ} 23^{\prime} 00^{\prime \prime}$ & $107^{\circ} 03^{\prime} 33^{\prime \prime}$ & $\mathrm{Ekm}$ & $006-\mathrm{AW}-2$ & 6,690 & - & $\mathrm{X}$ & -- \\
\hline 57 & 402002107050301 & SB00408607BDB & $40^{\circ} 20^{\prime} 02^{\prime \prime}$ & $107^{\circ} 05^{\prime} 03^{\prime \prime}$ & $\mathrm{Ekm}$ & $009-S-10$ & 6,905 & -- & $\mathrm{X}$ & -- \\
\hline 58 & 401926107053201 & SB00408618BCC & $40^{\circ} 19^{\prime} 26^{\prime \prime}$ & $107^{\circ} 05^{\prime} 32^{\prime \prime}$ & Ekm & $026-\mathrm{SP}-1$ & 7,080 & -- & $\mathrm{x}$ & -- \\
\hline 59 & 401951107045101 & SB00 408607DBA & $40^{\circ} 19^{\prime} 51^{\prime \prime}$ & $107^{\circ} 04^{\prime} 51^{\prime \prime}$ & $\mathrm{Ekm}$ & $026-\mathrm{SP}-2$ & 6,990 & -- & $\mathrm{X}$ & -- \\
\hline 60 & 401854107061201 & SB00408713DDC & $40^{\circ} 18^{\prime} 54^{\prime \prime}$ & $107^{\circ} 06^{\prime} 12^{\prime \prime}$ & $E \mathrm{~km}$ & $026-S P-3$ & 7,270 & -- & $\mathrm{X}$ & $\mathrm{X}$ \\
\hline 61 & 401828107065901 & SB00408724BCA & $40^{\circ} 18^{\prime} 28^{\prime \prime}$ & $107^{\circ} 06^{\prime} 59^{\prime \prime}$ & $\mathrm{Ekm}$ & 026-BRDH-1 & 7,530 & - & $\mathrm{X}$ & $X$ \\
\hline 62 & 401935107053201 & SB00408618BBB & $40^{\circ} 19^{\prime} 35^{\prime \prime}$ & $107^{\circ} 05^{\prime} 32^{\prime \prime}$ & $\mathrm{Ekm}$ & $026-79-4$ & 7,087 & - & $\mathrm{X}$ & - \\
\hline 64 & 401844107064101 & SB00408713CCD & $40^{\circ} 18^{\prime} 44^{\prime \prime}$ & $107^{\circ} 06^{\prime} 41^{\prime \prime}$ & $\mathrm{Ekm}$ & $026-79-7$ & 7,358 & -- & $\mathrm{X}$ & $\mathrm{X}$ \\
\hline 65 & 401955107043401 & SB00408607DAA & $40^{\circ} 19^{\prime} 55^{\prime \prime}$ & $107^{\circ} 04^{\prime} 34^{\prime \prime}$ & $\mathrm{Ekm}$ & $009-79-4$ & 7,003 & $\cdots$ & $\mathrm{X}$ & -- \\
\hline 66 & 402357106585101 & SB00508612 & $40^{\circ} 23^{\prime} 57^{\prime \prime}$ & $106^{\circ} 58^{\prime} 51^{\prime \prime}$ & $\mathrm{Ekm}$ & $008-A Y-1$ & 6,680 & -- & $\mathrm{X}$ & -- \\
\hline 67 & 402507107002901 & SB00508602 & $40^{\circ} 25^{\prime} 07^{\prime \prime}$ & $107^{\circ} 00^{\prime} 29^{\prime \prime}$ & Fis & $008-A U-2$ & 6,610 & -- & $\mathrm{X}$ & $\mathbf{X}$ \\
\hline 68 & 402504107002801 & SB00508602CCA & $40^{\circ} 25^{\prime} 04^{\prime \prime}$ & $107^{\circ} 00^{\prime} 28^{\prime \prime}$ & Fis & $008-A U-3$ & 6,608 & - & $\mathrm{X}$ & $\mathrm{X}$ \\
\hline 69 & 402501107003701 & SB00508602 & $40^{\circ} 25^{\prime} 01^{\prime \prime}$ & $107^{\circ} 00^{\prime} 27^{\prime \prime}$ & Fis & $008-\mathrm{AU}-4$ & 6,608 & -- & - & -- \\
\hline 70 & 402458107002701 & SB00508602 & $40^{\circ} 24^{\prime} 58^{\prime \prime}$ & $107^{\circ} 00^{\prime} 27^{\prime \prime}$ & Fis & $008-A U-5$ & 6,609 & -- & $\mathrm{X}$ & $\mathrm{X}$ \\
\hline 71 & 402456107002601 & SB00508602 & $40^{\circ} 24^{\prime} 56^{\prime \prime}$ & $107^{\circ} 00^{\prime} 26^{\prime \prime}$ & Fis & $008-A U-6$ & 6,610 & -- & $\mathrm{X}$ & $\mathrm{X}$ \\
\hline 72 & 402511106594501 & SB00508602DAB & $40^{\circ} 25^{\prime} 11^{\prime \prime}$ & $106^{\circ} 59^{\prime} 45^{\prime \prime}$ & Fis & $008-A W-3$ & 6,595 & -- & $\mathrm{X}$ & $\mathrm{x}$ \\
\hline 73 & 402245107052901 & SBO0508724ACA & $40^{\circ} 22^{\prime} 45^{\prime \prime}$ & $107^{\circ} 05^{\prime} 29^{\prime \prime}$ & Foi & $006-81-30$ & 6,780 & -- & $\mathrm{X}$ & $\mathrm{X}$ \\
\hline 74 & 402244107041601 & SB00508619AAC & $40^{\circ} 22^{\prime} 44^{\prime \prime}$ & $107^{\circ} 04^{\prime} 16^{\prime \prime}$ & Foi & $006-81-31$ & 6,805 & -- & $\mathrm{X}$ & - \\
\hline 75 & 402211107041801 & SB00508619DDC & $40^{\circ} 222^{\prime} 11^{\prime \prime}$ & $107^{\circ} 04^{\prime} 18^{\prime \prime}$ & Foi & $006-82-38$ & 7,080 & $\sim$ & $\mathrm{X}$ & $\mathrm{x}$ \\
\hline 76 & 402143107041801 & SB00508630ADC1 & $40^{\circ} 21^{\prime} 43^{\prime \prime}$ & $107^{\circ} 04^{\prime} 18^{\prime \prime}$ & Foi & $006-82-48$ & 7,300 & - & $\mathrm{x}$ & $\mathrm{x}$ \\
\hline 77 & 402143107041802 & SB00508630ADC2 & $40^{\circ} 21^{\prime} 43^{\prime \prime}$ & $107^{\circ} 04^{\prime} 18^{\prime \prime}$ & Foi & $006-82-48 A$ & 7,300 & -- & $\mathrm{x}$ & $\mathrm{X}$ \\
\hline 81 & 402220107100401 & SB00508720 & $40^{\circ} 22^{\prime} 20^{\prime \prime}$ & $107^{\circ} 10^{\prime} 04^{\prime \prime}$ & Gra & $M W-2$ & 7,230 & -- & $x$ & -- \\
\hline 82 & 402308107092501 & SB00508716 & $40^{\circ} 23^{\circ} 08^{\prime \prime}$ & $107^{\circ} 09^{\prime} 25^{\prime \prime}$ & Gra & $M W-3$ & 7,052 & -- & $\mathrm{X}$ & -- \\
\hline 83 & 402148107100601 & SB00508729 & $40^{\circ} 21^{\prime} 48^{\prime \prime}$ & $107^{\circ} 10^{\prime} 06^{\prime \prime}$ & Gra & PIT2 SPOIL & 7,670 & -- & $\mathrm{x}$ & -- \\
\hline 84 & 402124107104801 & SB00508729 & $40^{\circ} 21^{\prime} 24^{\prime \prime}$ & $107^{\circ} 10^{\prime} 48^{\prime \prime}$ & Gra & PIT5 SPOIL & 7,595 & -- & $\mathrm{X}$ & $\mathrm{X}$ \\
\hline 85 & 402204107180501 & SB00508830ACA I & $40^{\circ} 22^{\prime} 04^{\prime \prime}$ & $107^{\circ} 18^{\prime} 05^{\prime \prime}$ & Hyd & $\mathrm{LC}-2$ & 7,560 & -- & $x$ & -- \\
\hline 86 & 402204107180502 & SB00508830ACA2 & $40^{\circ} 22^{\prime} 04^{\prime \prime}$ & $107^{\circ} 18^{\prime} 05^{\prime \prime}$ & Hyd & UC- -2 & 7,560 & -- & $x$ & - \\
\hline 87 & 402203107174601 & SB00508830BAB1 & $40^{\circ} 22^{\circ} 03^{\prime \prime}$ & $107^{\circ} 17^{\prime} 46^{\prime \prime}$ & Hyd & $\mathrm{LC}-1$ & 7,475 & -- & $x$ & -- \\
\hline 88 & 402203107174602 & SB00508830BAB2 & $40^{\circ} 22^{\prime} 03^{\prime \prime}$ & $107^{\circ} 17^{\prime} 46^{\prime \prime}$ & Hyd & UC-1 & 7,475 & -- & $\mathrm{X}$ & -- \\
\hline 89 & 402154107175601 & SB00508830DBB & $40^{\circ} 21^{\prime} 54^{\prime \prime}$ & $107^{\circ} 17^{\prime} 56^{\prime \prime}$ & Hyd & LC -4 & 7,425 & -- & $X$ & -- \\
\hline 90 & 402144107174201 & SB00508830DDD & $40^{\circ} 21^{\prime} 44^{\prime \prime}$ & $107^{\circ} 17^{\prime} 42^{\prime \prime}$ & Hyd & $U C-3$ & 7,530 & -- & $\mathrm{X}$ & -- \\
\hline 91 & 402157107180901 & SB00508830BCA & $40^{\circ} 21^{\prime} 57^{\prime \prime}$ & $107^{\circ} 18^{\prime} 09^{\prime \prime}$ & Hyd & $\mathrm{LC}-3$ & 7,655 & -- & $\mathrm{X}$ & -- \\
\hline 92 & 402814107143901 & SB00608822BA & $40^{\circ} 28^{\prime} 14^{\prime \prime}$ & $107^{\circ} 14^{\prime} 39^{\prime \prime}$ & Hyd & UPDIP WELL & 6,380 & -- & $\mathrm{X}$ & -- \\
\hline 93 & 402815107142201 & SB00608815DD & $40^{\circ} 28^{\prime} 15^{\prime \prime}$ & $107^{\circ} 14^{\prime} 22^{\prime \prime}$ & Hyd & DWND I PWELL & 6,380 & -- & $x$ & -- \\
\hline 94 & 402854107070001 & SB00608714BDA & $40^{\circ} 28^{\prime} 54^{\prime \prime}$ & $107^{\circ} 07^{\circ} 00^{\prime \prime}$ & Med & CRUSH WELI & 6,400 & -- & $\mathrm{X}$ & $\mathrm{X}$ \\
\hline 95 & 402900107070401 & SB00608714BAC & $40^{\circ} 29^{\prime} 00^{\prime \prime}$ & $107^{\circ} 07^{\prime} 04^{\prime \prime}$ & Med & TRAIL WELL & 6,480 & -- & - & - \\
\hline
\end{tabular}


Table 5.--Ground-water site descriptions and availability of data collected by the U.S. Geological Survey

[Datum, altitude of land surface above sea level; USGS, U.S. Geological Survey; BLM, U.S. Bureau of Land Management; CONSOL, Consolidation Coal Company; X, data available; - , no data]

\begin{tabular}{|c|c|c|c|c|c|c|c|c|}
\hline $\begin{array}{l}\text { Map } \\
\text { number } \\
\text { (plate } \\
3 \text { ) }\end{array}$ & $\begin{array}{l}\text { Site identi- } \\
\text { fication in } \\
\text { COALDATA } \\
\text { data base }\end{array}$ & $\begin{array}{l}\text { Local well } \\
\text { number } \\
\text { (see fig. 2) }\end{array}$ & $\begin{array}{l}\text { Datum } \\
\text { (feet) }\end{array}$ & $\begin{array}{c}\text { Depth of well } \\
\text { below land } \\
\text { surface } \\
\text { (feet) }\end{array}$ & Owner & $\begin{array}{l}\text { A } \\
\text { Water- } \\
\text { level } \\
\text { data }\end{array}$ & $\begin{array}{l}\text { Jailabilit } \\
\text { Water- } \\
\text { quality } \\
\text { data }\end{array}$ & $\begin{array}{l}\text { ty of } \\
\text { Well-con- } \\
\text { struction } \\
\text { data }\end{array}$ \\
\hline 1 & 401500107363401 & SB00309109AAC & 7,150 & 8.00 & Hill Forbes & $\mathbf{X}$ & -- & $\mathrm{X}$ \\
\hline 2 & 401500107431401 & SB00309209BAD & 6,930 & 30.0 & Jin Leander & $\mathrm{X}$ & $\mathrm{X}$ & $x$ \\
\hline 3 & 401503107363901 & SB00309109ABAl & -- & 29.0 & J. Cooper & $\mathrm{X}$ & $\mathrm{x}$ & $\mathrm{x}$ \\
\hline 4 & 401503107460101 & SB00309312AAB & 6,810 & -- & Harry Kourlis & $\mathrm{X}$ & -- & $\mathrm{x}$ \\
\hline 5 & 401506107474401 & SB00309302CDC & 7,160 & -- & -2 & $\mathbf{X}$ & -- & $X$ \\
\hline 6 & 401508107485401 & SB00309303DCC & 7,360 & -- & -- & $\mathbf{X}$ & - & $\mathrm{X}$ \\
\hline 7 & 401510107483101 & SB00309310DCC & 7,340 & 198 & USGS & $\mathrm{X}$ & -- & $\mathrm{X}$ \\
\hline 8 & 401516107475000 & SB00309303CDB & -- & - & U.S. Government & $x$ & $\mathrm{X}$ & -- \\
\hline 9 & 401535107472001 & SB00309302ACC & 6,800 & - & - & $\mathrm{X}$ & -- & $\mathrm{x}$ \\
\hline 10 & 401539107472601 & SB00309302ACB & - & 30.0 & Colowyo Coal Co. & $x$ & $\mathrm{X}$ & $\mathrm{X}$ \\
\hline 11 & 401619107485401 & SB00309303CAC & 7,200 & 36.0 & -- & $\mathrm{X}$ & -- & $\mathrm{x}$ \\
\hline 12 & 401622107320201 & SB00409032BCD & 7,920 & 600 & -- & $\mathbf{X}$ & -- & $\mathrm{x}$ \\
\hline 13 & 401708107473301 & SB00409326CAD & 6,470 & 27.0 & Walter Mack & $\mathrm{X}$ & -- & $\mathrm{x}$ \\
\hline 14 & 401729107312701 & SB00409029ABA & 7,200 & -- & Phillips Petroleum & $\mathbf{X}$ & -- & $x$ \\
\hline 15 & 401741107453901 & SB00409230BBC & 6,300 & 30.0 & Jin Leander & $\mathrm{X}$ & -- & $\mathrm{x}$ \\
\hline 16 & 401801107470301 & SB00409323DAC & -- & 11.0 & Tom Wheeler & $\mathrm{x}$ & $x$ & $\mathrm{X}$ \\
\hline 17 & 401806107470901 & SB00409323DAB & - & 120 & Tom Wheeler & $\mathbf{X}$ & -- & $\mathbf{X}$ \\
\hline 18 & 401829107440501 & SB00409220ABC & 6,400 & 63.0 & Ton Moore & $X$ & -- & $\mathbf{X}$ \\
\hline 19 & 401833107333501 & SB00409113DCC & 7,270 & 500 & -- & $\mathrm{X}$ & -- & $\mathrm{X}$ \\
\hline 20 & 401838107411601 & SBD0 409223BBB & - & 2,930 & Texaco 0 il Co. & $x$ & -- & $\mathrm{x}$ \\
\hline 21 & 401847107314801 & SB00409017CAD & 6,830 & -- & H. Tuttle & $\mathrm{x}$ & $\cdots$ & $\mathbf{X}$ \\
\hline 22 & 401854107474901 & SB00409314CBD & 6,310 & 70.0 & Bill Gossard & $\mathrm{X}$ & $\mathrm{X}$ & $\mathbf{X}$ \\
\hline 23 & 401855107351401 & SB00409115DAD & 7,240 & -- & Phillips Petroleun & $\mathbf{X}$ & -- & $\mathrm{X}$ \\
\hline 24 & 401857107385201 & SB00409118DDB & 6,430 & -- & Al Camilletti & $\mathrm{X}$ & -- & $\mathrm{x}$ \\
\hline 25 & 401906107472901 & SB00409314BDD1 & - & 100 & Bill Gossard & $\mathrm{X}$ & $\mathrm{X}$ & $x$ \\
\hline 26 & 401943107392401 & SB00409212DCA & -- & 150 & Al Camilletti & $\mathrm{X}$ & -- & $\mathrm{x}$ \\
\hline 27 & 401944107352901 & SB00409110DDB & 7,020 & -- & Phillips Petroleum & $\mathrm{X}$ & -- & $X$ \\
\hline 28 & 401944107464901 & SB00409312CBC & -- & 30.0 & Bill Gossard & $\mathrm{X}$ & -- & $\mathrm{X}$ \\
\hline 29 & 401953107384701 & SB00409107DDB & 6,380 & 49.0 & H. Rakestraw & $\mathrm{X}$ & - & $\mathrm{X}$ \\
\hline 30 & 402001107481301 & SB00509335CDC & 6,310 & 70.0 & -- & $\mathrm{X}$ & -- & $\mathrm{X}$ \\
\hline 31 & 402013107374901 & SB00509132CDA & - & 380 & Al Camilletti & $\mathrm{X}$ & $\cdots$ & $\mathrm{X}$ \\
\hline 32 & 402019107385301 & SB00509 131DBB & 6,490 & -- & Moffat County & $X$ & -- & $\mathrm{X}$ \\
\hline 33 & 402049107325001 & SB00509 136AAA & 6,800 & 205 & Durham Livestock Co. & $\mathrm{x}$ & $\mathrm{x}$ & $\mathrm{X}$ \\
\hline 34 & 402116107361801 & SB00509 128DAC & -- & - & Myron Jeffcoat & $\mathrm{X}$ & - & $\mathrm{X}$ \\
\hline 35 & 402123107365301 & SB00509 $128 \mathrm{CAB}$ & -- & 12.0 & J.E. Carpenter & $\mathrm{X}$ & -- & $\mathrm{X}$ \\
\hline 36 & 402129107265201 & SB00509025BCD1 & 6,600 & 5.00 & L. Seeley & $\mathrm{X}$ & -- & $\mathrm{x}$ \\
\hline 37 & 402146107294601 & SB00509028ABA1 & 6,420 & 12.0 & D. Seeley & $\mathrm{X}$ & -- & $\mathrm{X}$ \\
\hline 38 & 402201107304601 & SB00509020DDB & 6,370 & 30.0 & Hamilton & $\mathrm{X}$ & -- & $\mathrm{X}$ \\
\hline 39 & 402203107304602 & SB00509020DAC2 & 6,360 & 460 & Gilbert Myers & $\mathrm{X}$ & $\cdots$ & $\mathrm{X}$ \\
\hline 40 & 402204107304501 & SB00509020DAC1 & 6,370 & 35.0 & Gilbert Myers & $\mathrm{X}$ & -- & $\mathrm{X}$ \\
\hline 41 & 402208107304201 & SB00509020DAC & 6,390 & 480 & Gilbert Myers & $\mathrm{X}$ & -- & $\mathrm{x}$ \\
\hline 42 & 402208107315201 & SB00509019DAC & - & 50.0 & Jacob Hamilton & $\mathrm{X}$ & - & $\mathrm{X}$ \\
\hline 43 & 402211107361901 & SB00509 121DAC & 6,260 & 53.0 & R.E. Southard & $\mathrm{X}$ & -- & $X$ \\
\hline 44 & 402211107363501 & SB00509121DBC & -- & 250 & Shirley Brown & $\mathrm{x}$ & -- & $\mathrm{X}$ \\
\hline 45 & 402211107363701 & SB00509121DBC & -- & 190 & Bill Kendall & $\mathrm{X}$ & - & $\mathrm{X}$ \\
\hline 46 & 402212107363402 & SB00509121DBC & -- & 25.0 & Shirley Brown & $\mathrm{x}$ & -- & $\mathrm{X}$ \\
\hline 47 & 402216107390801 & SB00509119CAB & 6,920 & 180 & Mead Brothers & $\mathrm{X}$ & $\mathrm{X}$ & $x$ \\
\hline 48 & 402244107385401 & SB00509119ABB & -- & 180 & Mead Brothers & $\mathrm{X}$ & -- & $\mathrm{X}$ \\
\hline 49 & 402301107445301 & SB00509208BCA & 6,240 & 205 & 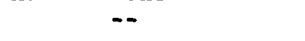 & $\mathrm{x}$ & $\mathrm{X}$ & -- \\
\hline 50 & 402316107282401 & SB00509015ADC1 & -- & -- & -- & -- & $x$ & Spring \\
\hline 51 & 402347107351900 & SB00509110DCD & -- & -- & -- & -- & $\mathrm{X}$ & Spring \\
\hline 52 & 402347107352201 & SB00509110DCD1 & - & -- & - & -- & $\mathrm{X}$ & Spring \\
\hline 53 & 402416107275801 & SB00509011BCB & -- & -- & -- & $\mathrm{X}$ & -- & -2 \\
\hline 54 & 402505107435501 & SB00509204BBB & 6,380 & 205 & - & $\mathrm{X}$ & $\mathrm{X}$ & - \\
\hline 55 & 402518107381801 & SB00509106ADA & 6,560 & - & Empire Energy Co. & $\mathrm{X}$ & -- & $\mathrm{X}$ \\
\hline
\end{tabular}


Table 5.--Ground-water site descriptions and availability of data collected by the U.S. GeologicaI Survey--Continued

\begin{tabular}{|c|c|c|c|c|c|c|c|c|}
\hline \multirow{2}{*}{$\begin{array}{l}\text { Map } \\
\text { number } \\
\text { (plate } \\
3)\end{array}$} & \multirow{2}{*}{$\begin{array}{l}\text { Site identi- } \\
\text { fication in } \\
\text { COALDATA } \\
\text { data base }\end{array}$} & \multirow[b]{2}{*}{$\begin{array}{c}\text { Local well } \\
\text { number } \\
\text { (see fig. } 2 \text { ) }\end{array}$} & \multirow[b]{2}{*}{$\begin{array}{l}\text { Datum } \\
\text { (feet) }\end{array}$} & \multirow{2}{*}{$\begin{array}{c}\text { Depth of well } \\
\text { below land } \\
\text { surface } \\
\text { (feet) }\end{array}$} & \multirow[b]{2}{*}{ Owner } & \multicolumn{3}{|c|}{ Availability of } \\
\hline & & & & & & $\begin{array}{l}\text { Water- } \\
\text { level } \\
\text { data }\end{array}$ & $\begin{array}{l}\text { Water- } \\
\text { quality } \\
\text { data }\end{array}$ & $\begin{array}{l}\text { Well-con- } \\
\text { struction } \\
\text { data }\end{array}$ \\
\hline 56 & 402520107292300 & SB00509004AAC1 & -- & -- & -- & $\mathbf{X}$ & -- & -- \\
\hline 57 & 402529107445901 & SB00609232CBC & 6,310 & 1,100 & -- & $\mathbf{X}$ & $\mathbf{X}$ & $\mathbf{x}$ \\
\hline 58 & 402541107420801 & SB00609234ACD & 6,100 & 60.0 & Elgen Lawrence & $\mathbf{X}$ & -- & $\mathrm{x}$ \\
\hline 59 & 402543107423201 & SB00609234BDC & 6,120 & 20.0 & Elgen Lawrence & $\mathbf{X}$ & -- & $\mathbf{X}$ \\
\hline 61 & 402558107421101 & SB00609234ABA & 6,240 & 832 & Elgen Lawrence & $\mathbf{X}$ & $\mathrm{X}$ & $\mathrm{X}$ \\
\hline 62 & 402613107390801 & SB00609136BBD1 & -- & 1,100 & $=$ & $\mathbf{X}$ & $\mathbf{X}$ & $\mathrm{X}$ \\
\hline 63 & 402627107381801 & SB00609131AAA & -- & 100 & Brasel and Simms & $\mathrm{X}$ & -- & $\mathrm{X}$ \\
\hline 64 & 402647107400600 & SB00609225BAD & -- & -- & -- & -- & $\mathbf{X}$ & -- \\
\hline 65 & 402647107400601 & SB00609225BAD1 & 6,610 & 600 & Haxton Iva & $\mathbf{X}$ & -- & -- \\
\hline 66 & 402649107382001 & SB00609130DAA & - & -- & W.R. Grace Co. & $\mathrm{x}$ & -- & $X$ \\
\hline 69 & 402718107333201 & SB00609112CCA1 & -- & - & $=$ & $\mathrm{X}$ & $\mathrm{X}$ & $\mathrm{x}$ \\
\hline 70 & 402722107310701 & SB00609020CDC & -- & 600 & Clyde Skidmore & $\mathbf{x}$ & -- & $\mathrm{x}$ \\
\hline 71 & 402734107301501 & SB00609021CBC & -- & 360 & Anton Knez & $\mathrm{X}$ & $\mathrm{X}$ & $\mathbf{x}$ \\
\hline 72 & 402738107422001 & SB00609222AAB & 6,400 & 800 & Ed Johnston & $\mathrm{X}$ & -- & $\mathbf{x}$ \\
\hline 73 & 402743107364702 & SB00609121CAB & 6,160 & 120 & Ed Loudy & $\mathbf{X}$ & -- & $\mathbf{X}$ \\
\hline 74 & 402744107364901 & SB00609121CAB & - & 700 & Ed Loudy & $\mathrm{x}$ & -- & $\mathrm{X}$ \\
\hline 75 & 402811107295301 & SB00609021BAB & -- & 40.0 & Mike Voloshin & $\mathbf{X}$ & -- & $\mathrm{X}$ \\
\hline 76 & 402812107271401 & SB00609014DCD1 & 6,580 & 182 & E.G. Deakins & $\mathrm{x}$ & $\mathbf{X}$ & $\mathrm{x}$ \\
\hline 77 & 402814107321101 & SB00609019BAB & 6,460 & 360 & Eugene Biskup & $\mathrm{x}$ & $\mathrm{X}$ & $\mathrm{x}$ \\
\hline 78 & 402814107490001 & SB00609315DBB & 6,120 & 425 & $\begin{array}{l}\text { U.S. Dept. of } \\
\text { Conservation. }\end{array}$ & $\mathbf{X}$ & $\mathbf{X}$ & $\mathrm{X}$ \\
\hline 79 & 402815107271101 & SB00609014DCD2 & 6,610 & 876 & E.G. Deakins & $\mathbf{X}$ & -- & $\mathrm{X}$ \\
\hline 80 & 402824107401001 & SB00609213BDA & 6,350 & 20.0 & John Salioko & $\mathrm{X}$ & -- & $\mathbf{X}$ \\
\hline 81 & 401735107344901 & SB00409126BAD1 & 8,123 & 115 & BLM & $\mathbf{X}$ & -- & $X$ \\
\hline 85 & 401752107310501 & SB00409021CBC & 7,000 & -- & CONSOL & -- & $\mathrm{x}$ & Spring \\
\hline 86 & 401613107325201 & SB00409031CAA & 7,640 & -- & CONSOL & -- & $\mathrm{X}$ & Spring \\
\hline 87 & 401646107314601 & SB00409029CAC & 7,768 & -- & -- & $\mathrm{x}$ & $\mathrm{x}$ & - \\
\hline 88 & 401726107312301 & SB00409029AAC1 & 7,240 & -- & -- & $\mathrm{x}$ & $\mathbf{X}$ & -- \\
\hline 89 & 401726107312302 & SB00409029AAC2 & 7,240 & -- & -- & $\mathrm{X}$ & $\mathrm{X}$ & -- \\
\hline 90 & 401654107312401 & SB00409029DCA & 7,560 & -- & -- & $\mathrm{X}$ & -- & -- \\
\hline 91 & 401551107312802 & SB00409032DCC2 & 8,408 & 221 & BLM & $\mathrm{X}$ & -- & $\mathrm{X}$ \\
\hline 92 & 401504107310801 & SB00409005DDC & 8,200 & -- & CONSOL & -- & $\mathrm{X}$ & Spring \\
\hline 93 & 401657107312901 & SB00409029DBD & 7,560 & -- & CONSOL & -- & $\mathrm{X}$ & Spring \\
\hline 94 & 401729107312301 & SB00409029AAB I & 7,160 & -- & CONSOL & -- & $\mathrm{X}$ & Spring \\
\hline 95 & 401730107312501 & SB00409029AAB2 & 7,160 & - & CONSOL & -- & $\mathrm{X}$ & Spring \\
\hline 96 & 401748107311102 & SB00409020DDA2 & 7,020 & -- & CONSOL & -- & $\mathrm{X}$ & Spring \\
\hline 97 & 401748107311101 & SB00409020DDA1 & 7,020 & 17.0 & CONSOL & $\mathrm{X}$ & $\mathrm{X}$ & $\mathrm{X}$ \\
\hline 98 & 401941107325901 & SB00409007CAA & 6,820 & -- & Almon Durham & $X$ & -- & $\mathrm{X}$ \\
\hline 99 & 401902107315101 & SB00409017BDB & 6,800 & -- & CONSOL & -- & $\mathrm{X}$ & Spring \\
\hline 100 & 401749107311201 & SB00409020DDA1 & 7,020 & 17.0 & CONSOL & $\mathrm{X}$ & - & $\mathrm{X}$ \\
\hline 101 & 401748107311501 & SB00409020DDA2 & 7,040 & 22.0 & CONSOL & $\mathrm{X}$ & $\mathrm{X}$ & $\mathrm{X}$ \\
\hline 102 & 401747107311401 & SB00409020DDA3 & 7,020 & 19.0 & -- & $\mathbf{x}$ & -- & $\mathrm{X}$ \\
\hline 103 & 401745107304401 & $\mathrm{SB00409021CDB}$ & 7,040 & 26.0 & CONSOL & $\mathrm{X}$ & -- & $\mathrm{x}$ \\
\hline 104 & 401747107311301 & SB00409020DDA4 & 7,020 & 25.7 & CONSOL & $\mathrm{X}$ & -- & $\mathrm{X}$ \\
\hline 105 & 401746107311301 & SB00409020DDA5 & 7,040 & 24.0 & CONSOL & $\mathrm{X}$ & $\mathrm{X}$ & $\mathbf{X}$ \\
\hline 106 & 401745107304301 & SB00409021CDB1 & 7,060 & 25.0 & CONSOL & $\mathrm{X}$ & $\mathrm{X}$ & $\mathrm{X}$ \\
\hline 107 & 401743107304101 & SB00409021CDD & 7,060 & 26.0 & CONSOL & $\mathrm{X}$ & -- & $\mathrm{x}$ \\
\hline 108 & 401742107304201 & SB00409021CDC & 7,060 & 26.0 & CONSOL & $\mathbf{X}$ & $\mathrm{X}$ & $x$ \\
\hline 109 & 401744107304501 & SB00409021CDB2 & 7,040 & 26.5 & -- & $\mathrm{x}$ & -- & $\mathrm{x}$ \\
\hline 110 & 401743107304601 & SB00409021CDB3 & 7,040 & 26.0 & CONSOL & $\mathrm{X}$ & $\mathrm{X}$ & $\mathrm{X}$ \\
\hline
\end{tabular}


Table 5.--Ground-water site descriptions and availability of data collected by the U.S. Geological Survey--Continued

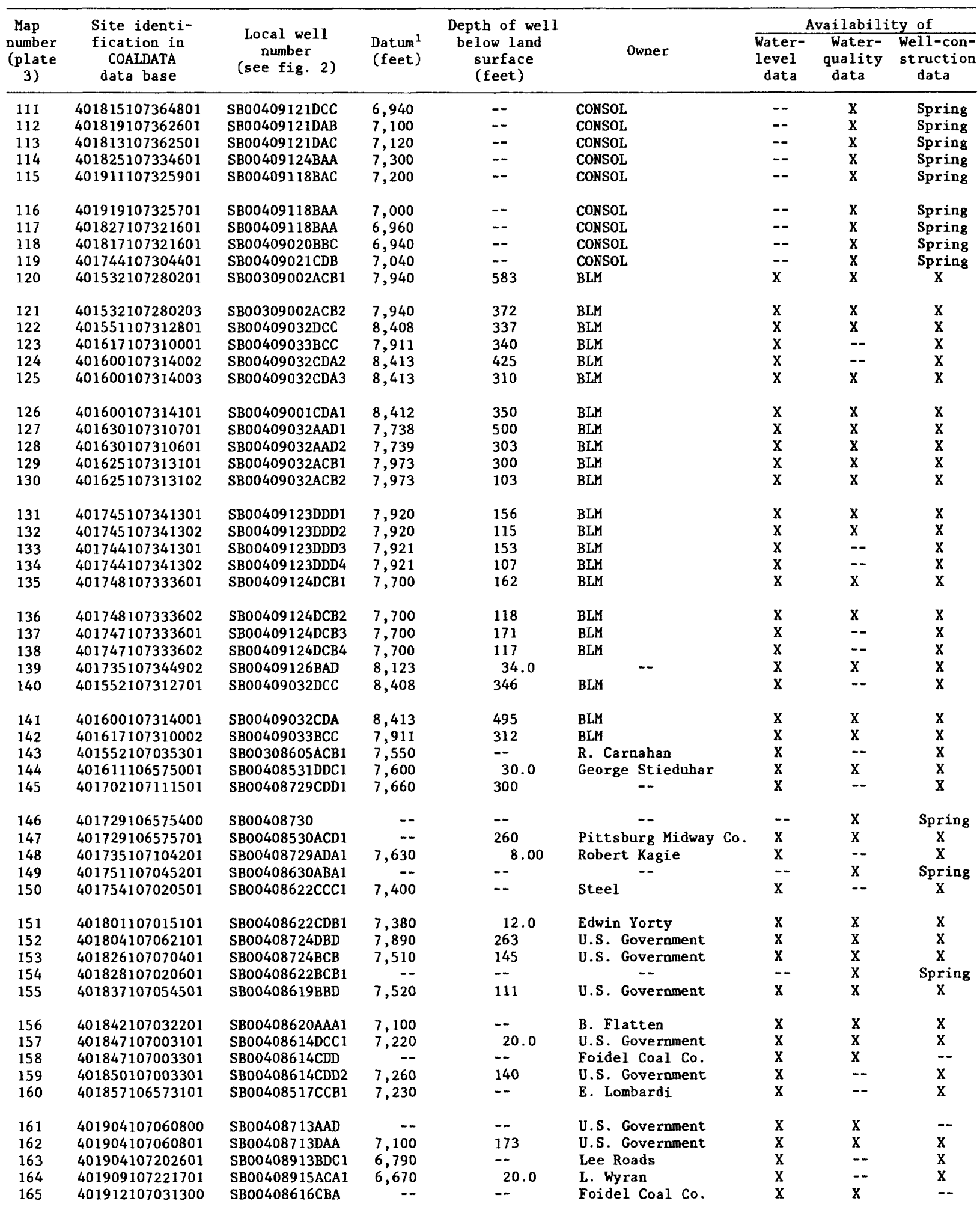


Table 5.--Ground-water site descriptions and availability of data collected by the U.S. Geological Survey--Continued

\begin{tabular}{|c|c|c|c|c|c|c|c|c|}
\hline \multirow{2}{*}{$\begin{array}{l}\text { Map } \\
\text { number } \\
\text { (plate } \\
\text { 3) }\end{array}$} & \multirow{2}{*}{$\begin{array}{l}\text { Site identi- } \\
\text { fication in } \\
\text { COALDATA } \\
\text { data base }\end{array}$} & \multirow[b]{2}{*}{$\begin{array}{c}\text { Local well } \\
\text { number } \\
\text { (see fig. 2) }\end{array}$} & \multirow[b]{2}{*}{$\begin{array}{l}\text { Datum } \\
\text { (feet) }\end{array}$} & \multirow{2}{*}{$\begin{array}{c}\text { Depth of well } \\
\text { below land } \\
\text { surface } \\
\text { (feet) }\end{array}$} & \multirow[b]{2}{*}{ Owner } & \multicolumn{3}{|c|}{ Availability of } \\
\hline & & & & & & $\begin{array}{l}\text { Water- } \\
\text { level } \\
\text { data }\end{array}$ & $\begin{array}{l}\text { Water- } \\
\text { quality } \\
\text { data }\end{array}$ & $\begin{array}{l}\text { Well-con- } \\
\text { struction } \\
\text { data }\end{array}$ \\
\hline 166 & 401912107031301 & SB00408616CBA & 7,020 & 122 & U.S. Government & $\mathbf{X}$ & $\mathrm{X}$ & $\mathrm{X}$ \\
\hline 167 & 401913107214301 & SB00408914BBDI & 6,740 & $\ldots$ & V. Preece & $\mathrm{X}$ & $\ldots$ & $\mathrm{X}$ \\
\hline 168 & 401914107244301 & SB00508931DDCl & 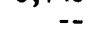 & 100 & Gail Coulverwel & $\mathrm{X}$ & $\ldots$ & $\mathrm{X}$ \\
\hline 169 & 401915107244301 & SBO0408917ABCl & - & 20.0 & H. Wynan & $\mathrm{X}$ & - & $\mathrm{X}$ \\
\hline 171 & 401922107050701 & SB00408618BDA & 7,160 & 180 & U.S. Government & $\mathbf{x}$ & $\mathrm{x}$ & $\mathrm{X}$ \\
\hline 172 & 401935106595401 & SB00408612CBD1 & 7,240 & 750 & USGS & $\mathrm{X}$ & $\mathrm{x}$ & $x$ \\
\hline 173 & 401939107091201 & SB004087 10CCD1 & 7,010 & 8.00 & K. Rhodes & $x$ & $\ldots$ & $\mathrm{X}$ \\
\hline 174 & 401941107002001 & SB00408611DCC & 7,490 & 1,030 & -2 & $\mathrm{X}$ & $\mathrm{X}$ & $\mathrm{X}$ \\
\hline 175 & 401941107222101 & SB00408910DBC1 & - & 210 & A.J. Klein & $\mathrm{X}$ & - & $\mathrm{x}$ \\
\hline 176 & 401950107103300 & SB00408709CBC & - & - & Dale Weldon & $\mathrm{X}$ & $\mathrm{X}$ & -- \\
\hline 177 & 401950107103301 & SB00408709CBC & 7,080 & 26.0 & ond & $\mathrm{X}$ & 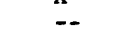 & $\mathrm{X}$ \\
\hline 179 & 402005107200101 & SB00408912ABD1 & - & -- & -2 & $\cdots$ & $\mathrm{x}$ & Spring \\
\hline 180 & 402013107245201 & SB00508931DDC 1 & -- & -- & -- & $\mathrm{X}$ & $\mathrm{X}$ & -- \\
\hline 181 & 402015107014501 & SB00408610BAD & 6,940 & 100 & U.S. Government & $\mathrm{x}$ & $\mathrm{X}$ & $\mathrm{X}$ \\
\hline 182 & 402015107015500 & SB00408610BAD & -- & -- & Foide1-Midway Co. & $\mathrm{x}$ & $\mathrm{X}$ & $\ldots$ \\
\hline 183 & 402040106591001 & SB00508636CAC1 & 7,160 & 716 & USGS & $\mathrm{X}$ & - & $\mathbf{X}$ \\
\hline 184 & 402041107013301 & SB00508634CAB1 & 6,920 & 200 & D. Hayes & $\mathrm{X}$ & $\mathrm{X}$ & $\mathbf{x}$ \\
\hline 185 & 402048107203201 & SB00508923CCC & -. & -- & - & $\mathrm{x}$ & $\mathrm{x}$ & $\ldots$ \\
\hline 186 & 402056106590901 & SB00508636BDD1 & 7,080 & 560 & Perley Green & $\mathrm{X}$ & $\mathrm{x}$ & $\mathrm{X}$ \\
\hline 187 & 402106107011801 & SB00508622DCC1 & 6,820 & $\ldots$ & William Rogers & $\mathrm{X}$ & - & $\mathrm{x}$ \\
\hline 188 & 402108107220301 & SB00508923CCC & 7,360 & 280 & -2 & $\mathrm{x}$ & $\mathrm{X}$ & $\mathrm{X}$ \\
\hline 189 & 402112107050801 & SB00508630CCC1 & -0 & 350 & Dale Kemeer & $\mathrm{X}$ & $\mathrm{X}$ & $\mathrm{X}$ \\
\hline 190 & 402113106590501 & SB00508636BAA2 & 7,080 & 750 & Perley Greene & $x$ & $\mathrm{x}$ & $\mathbf{x}$ \\
\hline 191 & 402114107034300 & SB00508629CDD1 & 6,820 & 126 & U.S. Government & $\mathbf{x}$ & $\mathrm{X}$ & $x$ \\
\hline 192 & 402114107034301 & SB00508629CDD2 & 6,820 & 266 & U.S. Government & $\mathrm{X}$ & $\mathrm{X}$ & $\mathrm{X}$ \\
\hline 196 & 402149107013801 & SB00508627BCA1 & 6,840 & -- & Robert George & $\mathbf{x}$ & $\mathrm{x}$ & $\mathbf{x}$ \\
\hline 197 & 402156107015201 & SB00508627BBC1 & 6,810 & 14.0 & Robert George & $\mathrm{X}$ & $\mathrm{X}$ & $\mathbf{x}$ \\
\hline 198 & 402157106583801 & SB00508625AACl & -- & 20.0 & Robert McKune & $\mathrm{X}$ & $\cdots$ & $\mathrm{X}$ \\
\hline 199 & 402158106570701 & SB00408529BAC1 & 7,060 & 40.0 & Giacomo Camilletti & $\mathrm{X}$ & $\cdots$ & - \\
\hline 200 & 402202107022101 & SB00508628ABB 1 & 6,800 & 14.0 & Vaughn Hockaday & $\hat{x}$ & $\mathrm{X}$ & $\mathrm{X}$ \\
\hline 201 & 402202107160801 & SB00508809CDB 1 & 6,750 & 15.0 & H. Green & $\mathrm{x}$ & - & $\mathrm{X}$ \\
\hline 202 & 402204107012201 & SB00508627BAA & 6,820 & 18.0 & U.S. Government & $x$ & $x$ & $\mathrm{X}$ \\
\hline 203 & 402204107022801 & SB00508628BAA 1 & - & - & 0.0. & $\hat{x}$ & $\hat{x}$ & - \\
\hline 204 & 402205107013201 & SB00508627BAB 1 & 6,800 & 14.0 & Don Hinkle & $\mathrm{X}$ & $\mathrm{X}$ & $\mathrm{X}$ \\
\hline 205 & 402209107023101 & SB00508621CDD & 6,840 & 282 & U.S. Government & $\mathrm{X}$ & $\mathrm{X}$ & $\mathrm{X}$ \\
\hline 206 & 402212107011801 & SB00508622DCCl & -- & -- & -- & $\mathrm{X}$ & $\mathbf{x}$ & - \\
\hline 207 & 402213107024401 & SB00508621CCA1 & $\ldots$ & -- & - & $\ldots$ & $\mathrm{X}$ & Spring \\
\hline 208 & 402217107113601 & SB00508719CDA2 & 7,380 & 18.0 & Leonard Yoast & $\mathbf{X}$ & - & -- \\
\hline 209 & 402221107005201 & SB00508622DAD1 & $\ldots$ & -- & $\ldots$ & -- & $x$ & Spring \\
\hline 210 & 402222107113001 & SB005087 19CDA1 & 7,370 & 90.0 & Leonard Yoast & $\mathrm{X}$ & $\mathrm{X}$ & $\mathrm{X}$ \\
\hline 211 & 402231107111601 & SB00508719ABA2 & 7,340 & 62.0 & Peabody Coal Co. & $\mathbf{x}$ & $\mathrm{x}$ & $\mathrm{X}$ \\
\hline 212 & 402231107111602 & SB00508719ABA1 & 7,320 & 58.5 & Peabody Coal Co. & $\mathrm{X}$ & $\mathrm{x}$ & $\mathrm{X}$ \\
\hline 213 & 402231107111603 & SBO05087 19DBA1 & 7,580 & 133 & Peabody Coa 1 Co. & $\mathrm{x}$ & $\ldots$ & $\mathrm{X}$ \\
\hline 214 & 402231107111604 & SB005087 19DBA2 & 7,580 & 146 & Peabody Coal Co. & $\mathrm{X}$ & -- & $\mathrm{x}$ \\
\hline 215 & 402231107111605 & SB00508730ABA 1 & 7,740 & 9.50 & Peabody Coal Co. & $\mathrm{X}$ & -- & $\mathrm{x}$ \\
\hline 216 & 402231107111606 & SB00508730ABA2 & 7,740 & 26.3 & Peabody Coal Co. & $\mathrm{x}$ & $\ldots$ & $x$ \\
\hline 217 & 402231107111607 & SB00508729BBA1 & 7,980 & 65.0 & Peabody Coal Co. & $\mathrm{X}$ & -- & $\mathrm{X}$ \\
\hline 218 & 402231107111609 & SB00508720CBA1 & 7,800 & 48.0 & Peabody Coal Co. & $x$ & -- & $\mathrm{X}$ \\
\hline 219 & 402231107111611 & SB00508720BBA 1 & 7,700 & 27.0 & Peabody Coal co. & $\mathrm{X}$ & -- & $\mathrm{X}$ \\
\hline 220 & 402231107111612 & SB00508720BBA2 & 7,700 & 37.4 & Peabody Coal Co. & $\mathrm{x}$ & $x$ & $\mathrm{X}$ \\
\hline
\end{tabular}


Table 5.--Ground-water site descriptions and availability of data collected by the U.S. Geological Survey--Continued

\begin{tabular}{|c|c|c|c|c|c|c|c|c|}
\hline \multirow{2}{*}{$\begin{array}{l}\text { Map } \\
\text { number } \\
\text { (plate } \\
\text { 3) }\end{array}$} & \multirow{2}{*}{$\begin{array}{l}\text { Site identi- } \\
\text { fication in } \\
\text { COALDATA } \\
\text { data base }\end{array}$} & \multirow[b]{2}{*}{$\begin{array}{c}\text { Local well } \\
\text { number } \\
\text { (see fig. 2) }\end{array}$} & \multirow[b]{2}{*}{$\begin{array}{l}\text { Datum } \\
\text { (feet) }\end{array}$} & \multirow{2}{*}{$\begin{array}{l}\text { Depth of well } \\
\text { below land } \\
\text { surface } \\
\text { (feet) }\end{array}$} & \multirow[b]{2}{*}{ Owner } & \multicolumn{3}{|c|}{ Availability of } \\
\hline & & & & & & $\begin{array}{l}\text { Water- } \\
\text { level } \\
\text { data }\end{array}$ & $\begin{array}{l}\text { Water- } \\
\text { quality } \\
\text { data }\end{array}$ & $\begin{array}{l}\text { Well-con- } \\
\text { struction } \\
\text { data }\end{array}$ \\
\hline 221 & 402231107111613 & SB00508717CBC2 & 7,540 & 85.7 & Peabody Coal Co. & $\mathrm{X}$ & -- & $X$ \\
\hline 222 & 402231107111614 & SB00508717CBC1 & 7,540 & 101 & Peabody Coal Co. & $\mathrm{X}$ & -- & $\mathrm{X}$ \\
\hline 223 & 402231107111615 & SB00508717BCC2 & 7,400 & 79.5 & Peabody Coal Co. & $\mathrm{X}$ & -- & $\mathrm{X}$ \\
\hline 224 & 402231107111616 & SB00508717BCC1 & 7,400 & 104 & Peabody Coal Co. & $\mathrm{X}$ & $\mathrm{X}$ & $\mathrm{X}$ \\
\hline 225 & 402231107111617 & SB00508717ADC1 & 7,560 & 90.0 & Peabody Coal Co. & $\mathrm{X}$ & $\mathrm{X}$ & $\mathrm{X}$ \\
\hline 226 & 402231107111618 & $\mathrm{SB} 00508717 \mathrm{ADC} 2$ & 7,560 & 109 & Peabody Coal Co. & $\mathrm{X}$ & - & $\mathrm{X}$ \\
\hline 227 & 402231107111619 & SB00508717DAC1 & 7,760 & 25.0 & Peabody Coal Co. & $\mathrm{X}$ & -- & $\mathrm{X}$ \\
\hline 228 & 402231107111620 & SB00508717DAC2 & 7,760 & 43.0 & Peabody Coal Co. & $\mathbf{x}$ & - & $\mathrm{X}$ \\
\hline 229 & 402231107111630 & SB00508719ABB1 & 7,300 & 47.0 & Peabody Coal Co. & $\mathrm{X}$ & $\mathrm{X}$ & $\mathrm{X}$ \\
\hline 230 & 402231107111631 & SB00508719ABB2 & 7,300 & 47.0 & Peabody Coal Co. & $\mathrm{X}$ & $\mathbf{x}$ & $\mathrm{X}$ \\
\hline 231 & 402231107111632 & SB00508719ABB3 & 7,300 & 54.0 & Peabody Coal Co. & $\mathrm{X}$ & $X$ & $\mathrm{X}$ \\
\hline 232 & 402231107111633 & SB00508719ABB4 & 7,300 & 54.0 & Peabody Coal Co. & $\mathrm{X}$ & $\mathrm{X}$ & $\mathrm{x}$ \\
\hline 233 & 402231107111634 & SB005087 19DBA3 & 7,580 & 135 & Peabody Coal Co. & $\mathrm{X}$ & $\mathbf{X}$ & $\mathrm{X}$ \\
\hline 234 & 402231107111635 & SB005087 19DBA4 & 7,580 & 135 & Peabody Coal Co. & $\mathrm{X}$ & -- & $\mathrm{X}$ \\
\hline 235 & 402231107111636 & SB005087 19DBA5 & 7,580 & 151 & Peabody Coal Co. & $\mathbf{X}$ & $\mathbf{x}$ & $\mathrm{x}$ \\
\hline 236 & 402231107111637 & SB00508719DBA6 & 7,580 & 151 & Peabody Coal Co. & $\mathrm{X}$ & $\mathbf{x}$ & $\mathrm{X}$ \\
\hline 237 & 402231107111638 & SB005087 19DBA7 & 7,580 & 170 & Peabody Coal Co. & $\mathbf{X}$ & -- & $\mathrm{X}$ \\
\hline 238 & 402231107111646 & SB00508718CAC1 & 7,200 & 39.1 & USGS & $\mathrm{X}$ & $\mathrm{X}$ & $\mathrm{X}$ \\
\hline 239 & 402231107111647 & SB00508718CAC2 & 7,200 & 29.4 & USGS & $\mathbf{x}$ & - & $\mathrm{x}$ \\
\hline 240 & 402231107111648 & SB00508719BAA1 & 7,240 & 44.0 & USGS & $\mathrm{X}$ & -- & $\mathbf{X}$ \\
\hline 241 & 402231107111649 & SB00508719BAA2 & 7,240 & 59.0 & USGS & $\mathrm{X}$ & -- & $\mathrm{X}$ \\
\hline 242 & 402231107111650 & SB00508719BAA3 & 7,240 & 45.0 & USGS & $\mathrm{X}$ & -- & $\mathrm{X}$ \\
\hline 243 & 402231107111651 & SB005087 19CCB1 & 7,360 & 40.0 & USGS & $\mathrm{X}$ & - & $\mathrm{X}$ \\
\hline 244 & 402231107111652 & SB00508719CCB2 & 7,360 & 45.0 & USGS & $\mathrm{X}$ & 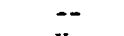 & $\mathrm{X}$ \\
\hline 245 & 402231107111653 & SB00508719CCB3 & 7,360 & 20.0 & USGS & $x$ & $\mathrm{X}$ & $\mathrm{X}$ \\
\hline 246 & 402231107111654 & SB00508719CCB 4 & 7,360 & 20.0 & USGS & $\mathrm{X}$ & -- & $x$ \\
\hline 247 & 402231107111655 & SB00508719CCA1 & 7,360 & 50.0 & USGS & $\mathrm{X}$ & -- & $\mathrm{X}$ \\
\hline 248 & 402231107111656 & SB00508719CDC1 & 7,360 & 40.0 & USGS & $\mathbf{X}$ & -- & $x$ \\
\hline 249 & 402231107111657 & SB00508719CDC2 & 7,360 & 40.0 & USGS & $\mathrm{X}$ & $\mathrm{X}$ & $x$ \\
\hline 250 & 402231107111658 & SBO0508719CDC3 & 7,360 & 30.0 & USGS & $\mathbf{X}$ & -- & $\mathrm{X}$ \\
\hline 251 & 402232107234201 & SB00508920ACD1 & 7,820 & 640 & BLM & $\mathrm{X}$ & $\mathrm{x}$ & $\mathrm{X}$ \\
\hline 252 & 402236107025301 & SB00508621BCC & 6,760 & 198 & U.S. Government & $\mathrm{X}$ & $\mathrm{X}$ & $\mathrm{X}$ \\
\hline 253 & 402239107040301 & SB00508620BCB & 6,820 & 30.0 & U.S. Government & $\mathrm{X}$ & $\mathrm{x}$ & $\mathrm{X}$ \\
\hline 254 & 402244107165001 & SB00508820ACA1 & - & 49.0 & -- & $\mathrm{X}$ & $\mathrm{X}$ & -- \\
\hline 255 & 402244107165002 & SB00508820DAB1 & $\cdots$ & - & -- & $\mathrm{X}$ & -- & -- \\
\hline 256 & 402250107151801 & $\mathrm{SB} 00508822 \mathrm{BBCl}$ & -- & -- & -- & $\mathrm{X}$ & $\mathrm{X}$ & -- \\
\hline 257 & 402250107151802 & SB00508822BBC2 & 7,060 & 235 & -- & $\mathrm{X}$ & -- & $\mathrm{X}$ \\
\hline 258 & 402257107015301 & SB00508621AAA & 6,700 & 207 & U.S. Government & $\mathrm{X}$ & $\mathrm{X}$ & $\mathbf{X}$ \\
\hline 259 & 402303107215701 & SB00508915CAB & 7,400 & 440 & -- & $x$ & $X$ & $\mathrm{X}$ \\
\hline 260 & 402316107182301 & SB00508913ACC & 7,000 & 720 & -- & $x$ & $\mathrm{x}$ & $\mathrm{X}$ \\
\hline 261 & 402327106590000 & SB00508613ACC & -- & - & Foidel-Midway Co. & $X$ & $\mathrm{X}$ & -- \\
\hline 262 & 402327106590001 & SB00508613ACC & 6,680 & 237 & U.S. Government & $X$ & $X$ & $\mathrm{X}$ \\
\hline 263 & 402327107161301 & $\mathrm{SB} 00508816 \mathrm{CAB} 1$ & - & 62.0 & -- & $\mathrm{x}$ & $X$ & -- \\
\hline 264 & 402334106574601 & SB005085 18ACA1 & 6,800 & 80.0 & Wegener & $\mathrm{X}$ & -- & $\mathbf{X}$ \\
\hline 265 & 402336106574400 & SB00508518ACA & 6,780 & 105 & Truman Yowel1 & $X$ & $\mathrm{X}$ & $\mathrm{X}$ \\
\hline 266 & 402344107195001 & SB00508912CCD1 & -- & -- & P. Barns & $X$ & $X$ & $\mathrm{X}$ \\
\hline 267 & 402346106590000 & SB00508613ABB & -- & -- & Foidel-Midway Co. & $\mathrm{X}$ & $\mathrm{X}$ & -- \\
\hline 268 & 402346106590001 & SB00508613ABB & 6,660 & 100 & U.S. Government & $\mathrm{X}$ & $\mathrm{X}$ & $\mathrm{X}$ \\
\hline 269 & 402356107171701 & SB00508808CDC & 6,780 & 80.0 & $\begin{array}{l}\text { U.S. Dept. of } \\
\text { Conservation. }\end{array}$ & $X$ & $\mathrm{X}$ & $\mathrm{X}$ \\
\hline 270 & 402414106580701 & $S B 00508507 C B C 1$ & 6,680 & 180 & - & $\mathrm{X}$ & $\cdots$ & $\mathrm{x}$ \\
\hline 271 & 402414106585701 & SB00508612DBB1 & -- & - & $-\infty$ & $\mathrm{X}$ & $\mathrm{X}$ & -- \\
\hline 272 & 402415107184401 & SB00508807CBB1 & 6,680 & 85.0 & Marvin D. Barnes & $\mathrm{X}$ & -- & $\mathrm{X}$ \\
\hline 273 & 402430107070801 & SB00508711BDB1 & -- & -- & -- & $\mathrm{X}$ & -- & -- \\
\hline 274 & 402438106572001 & SB00508508BBC1 & 6,960 & -- & Streeter & $\mathrm{X}$ & -- & $\mathrm{X}$ \\
\hline 275 & 402440106591001 & SB00508612BAC & - & -- & - & $\mathrm{X}$ & $\mathrm{X}$ & -- \\
\hline
\end{tabular}


Table 5.--Ground-water site descriptions and availability of data collected by the U.S. Geological Survey--Continued

\begin{tabular}{|c|c|c|c|c|c|c|c|c|}
\hline \multirow{2}{*}{$\begin{array}{l}\text { Map } \\
\text { number } \\
\text { (plate } \\
3 \text { ) }\end{array}$} & \multirow{2}{*}{$\begin{array}{l}\text { Site identi- } \\
\text { fication in } \\
\text { COALDATA } \\
\text { data base }\end{array}$} & \multirow[b]{2}{*}{$\begin{array}{c}\text { Local well } \\
\text { number } \\
\text { (see fig. 2) }\end{array}$} & \multirow[b]{2}{*}{$\begin{array}{l}\text { Datum }{ }^{1} \\
\text { (feet) }\end{array}$} & \multirow{2}{*}{$\begin{array}{c}\text { Depth of well } \\
\text { below land } \\
\text { surface } \\
\text { (feet) }\end{array}$} & \multirow[b]{2}{*}{ Owner } & \multicolumn{3}{|c|}{ Availability of } \\
\hline & & & & & & $\begin{array}{l}\text { Water- } \\
\text { level } \\
\text { data }\end{array}$ & $\begin{array}{l}\text { Water- } \\
\text { quality } \\
\text { data }\end{array}$ & $\begin{array}{l}\text { Well-con- } \\
\text { struction } \\
\text { data }\end{array}$ \\
\hline 276 & 402459107154901 & SB00508804DBC1 & -- & 80.0 & G.A. Bowen & $\mathrm{X}$ & $\mathrm{X}$ & $\mathbf{X}$ \\
\hline 277 & 402514106561501 & SB00508505ADD 1 & -- & 91.0 & J.T. Kelton & $\mathrm{X}$ & $\mathrm{X}$ & $\mathbf{x}$ \\
\hline 278 & 402514107065001 & SB00508702ACC1 & -- & -- & -- & $\mathrm{x}$ & -- & -- \\
\hline 279 & 402514107065002 & SB00508702ACC2 & -- & -- & -- & $\mathrm{X}$ & -- & -- \\
\hline 281 & 402517106590701 & SB00508601BDA1 & 6,600 & -- & -- & $\mathrm{x}$ & -- & -- \\
\hline 282 & 402526107231601 & SB00508904BBAl & 6,920 & 80.0 & Otis Lyons & $\mathrm{X}$ & $\mathrm{X}$ & $\mathrm{X}$ \\
\hline 283 & 402535106582301 & SB00608531CCC1 & 6,580 & -- & V. Lazer & $\mathrm{X}$ & - & $\mathbf{x}$ \\
\hline 284 & 402535106582501 & SB00608531CCCI & 6,600 & -- & Adams & $\mathrm{X}$ & -- & $\mathrm{X}$ \\
\hline 285 & 402535107234401 & SB00608932DDB & 7,060 & -- & Otis Lyons & $\mathrm{X}$ & -- & $\mathrm{x}$ \\
\hline 286 & 402545107073901 & SB00608734DDB1 & -- & -- & -- & $\mathrm{X}$ & $\mathbf{x}$ & -- \\
\hline 287 & 402600107160001 & SB00608833DBB & 6,500 & 205 & $\begin{array}{l}\text { U.S. Dept. of } \\
\text { Conservation. }\end{array}$ & $\mathrm{X}$ & $\mathrm{X}$ & $\mathrm{x}$ \\
\hline 288 & 402609107124801 & SB00608836BDC 1 & -- & 60.0 & William Coley & $\mathrm{x}$ & $\mathrm{X}$ & $\mathbf{x}$ \\
\hline 289 & 402614107125001 & SB00608836BDB1 & 6,650 & 66.0 & Allen Codey & $\mathrm{X}$ & -- & $\mathbf{X}$ \\
\hline 290 & 402620107072924 & SB00608734DDA1 & 6,800 & 52.0 & Peabody Coal Co. & $\mathrm{x}$ & -- & $\mathrm{X}$ \\
\hline 291 & 402620107072925 & SB00608734DAA1 & 6,800 & 25.0 & Peabody Coal Co. & $\mathbf{X}$ & -- & $\mathbf{X}$ \\
\hline 292 & 402620107072926 & SB00608734DAD1 & 6,760 & 64.0 & Peabody Coal Co. & $\mathbf{x}$ & -- & $\mathbf{x}$ \\
\hline 293 & 402620107072928 & SB00608734ADA1 & 6,760 & 50.5 & Peabody Coal Co. & $\mathbf{X}$ & $\mathbf{X}$ & $\mathrm{X}$ \\
\hline 294 & 402620107072929 & SB00608734ADA2 & 6,740 & 43.5 & Peabody Coal Co. & $\mathrm{X}$ & $\mathrm{X}$ & $\mathrm{X}$ \\
\hline 295 & 402620107072930 & SB00608734ADB3 & 6,720 & 38.5 & Peabody Coal Co. & $\mathrm{X}$ & $\mathrm{x}$ & $\mathrm{X}$ \\
\hline 296 & 402620107072932 & SB00608734ACA1 & 6,640 & 143 & Peabody Coal Co. & $\mathbf{X}$ & $\mathrm{X}$ & $\mathbf{x}$ \\
\hline 297 & 402620107072933 & SB00608734ACA2 & 6,640 & 141 & Peabody Coal Co. & $\mathrm{X}$ & $\mathbf{x}$ & $\mathrm{X}$ \\
\hline 298 & 402620107072935 & SB00508703DAC1 & 6,840 & 260 & Peabody Coal Co. & $\mathrm{X}$ & $\mathrm{X}$ & $\mathrm{X}$ \\
\hline 299 & 402620107072939 & SB00608722DDD1 & -- & -- & -- & $\mathrm{X}$ & $\mathrm{x}$ & -- \\
\hline 300 & 402620107072950 & SB00608734DDB 1 & 6,740 & 261 & Peabody Coal Co. & $\mathrm{X}$ & $\mathbf{X}$ & $\mathrm{X}$ \\
\hline 301 & 402620107072951 & SB00608734DDB2 & 6,740 & 78.0 & Peabody Coal Co. & $\mathbf{x}$ & $\mathbf{x}$ & $\mathrm{x}$ \\
\hline 305 & 402620107072956 & SB00608734BAB 1 & -- & -- & - & $\mathrm{X}$ & $\mathbf{x}$ & -- \\
\hline 306 & 402620107072958 & SB00608734 & -- & -- & Peabody Coal Co. & $\mathrm{X}$ & $\mathbf{x}$ & -- \\
\hline 307 & 402627107115900 & SB00608731BBB & -- & -- & Jim Rowley & $\mathbf{X}$ & $\mathbf{x}$ & -- \\
\hline 308 & 402627107115901 & SB00608731BBB & 6,580 & -- & Jim Rowley & $x$ & -- & $\mathbf{X}$ \\
\hline 309 & 402633107080101 & SB00608727DCA & $=$ & 10.0 & Seneca Coal Co, & $\mathrm{X}$ & $\mathrm{X}$ & $\mathrm{x}$ \\
\hline 310 & 402641107090201 & SB00608728DCA 1 & 6,600 & -- & H. Rhodes & $x$ & -- & $x$ \\
\hline 311 & 312643107153301 & SB00608828DAD1 & 6,460 & 160 & Cross Mountain Ranch & $x$ & $\mathrm{x}$ & $\mathbf{x}$ \\
\hline 312 & 402646106585501 & SB00608625DBC1 & 6,550 & 13.0 & Arnold Lieske & $x$ & -- & $x$ \\
\hline 313 & 402647106585000 & SB00608625DBD & - & -- & Arnold Lieske & $\mathrm{X}$ & $\mathbf{x}$ & -- \\
\hline 314 & 402647106585001 & SB00608625DBD & 6,580 & 12.0 & Arnold Lieske & $x$ & -- & $\mathrm{X}$ \\
\hline 315 & 402709106591201 & SB00608625BDB 1 & 6,590 & 116 & Dan Bede11 & $\mathbf{x}$ & $x$ & $\mathrm{x}$ \\
\hline 316 & 402709107252501 & SB00608930BAB 1 & - & -- & - & $\mathrm{X}$ & $\mathrm{x}$ & -- \\
\hline 317 & 402710106591401 & SB00608625BAC1 & 6,600 & 116 & Bedells & $\mathrm{x}$ & -- & $\mathbf{x}$ \\
\hline 318 & 402738107011300 & SB00608622DBC & - & -- & -- & $x$ & $\mathrm{X}$ & -- \\
\hline 319 & 402738107011301 & SB00608622DBC & 6,580 & -- & -- & -- & - & Spring \\
\hline 320 & 402804107095201 & SB00608720AAC 1 & 6,500 & -- & Beryl Rienks & $\mathrm{x}$ & -- & $\mathrm{x}$ \\
\hline 321 & 402809106595501 & SB00608623ABA1 & 6,600 & 260 & Wyman & $\mathrm{x}$ & -- & $\mathrm{x}$ \\
\hline 322 & 402815107003001 & SB00608623BBA1 & 6,490 & 150 & E.C. Black & $\mathrm{X}$ & -- & $X$ \\
\hline 323 & 401710107562800 & SB00409428DBB & -- & -- & W.R. Grace Co. & -- & $\mathbf{X}$ & -- \\
\hline 324 & 401759107555400 & SB00409422CBC & -- & -- & W.R. Grace Co. & -- & $\mathrm{X}$ & -- \\
\hline 325 & 401810107553200 & SB00409422BDD & -- & -- & W.R. Grace Co. & -- & $\mathrm{x}$ & -- \\
\hline 326 & 402120106535201 & SB00508526CCC1 & 6,990 & 105 & Don Zoller & -- & $\mathrm{X}$ & $\mathrm{X}$ \\
\hline 327 & 402146106543401 & SB00508527ACCl & 7,100 & 17.0 & W. Whitecotton & $\mathbf{x}$ & $\cdots$ & $\mathrm{X}$ \\
\hline 328 & 329157106543101 & SB00508527ABC1 & 7,110 & 17.0 & W. Whitecotton & $\mathbf{x}$ & - & $\mathbf{X}$ \\
\hline 329 & 402309106520801 & SB00508513DDB & 6,920 & 15.0 & -- & $\mathrm{X}$ & -- & -- \\
\hline 330 & 402321106534601 & SB $00508514 \mathrm{CBA}$ & 7,160 & -- & -- & $\mathrm{X}$ & -- & -- \\
\hline
\end{tabular}


Table 5.--Ground-water site descriptions and availability of data collected by the U.S. Geological Survey--Continued

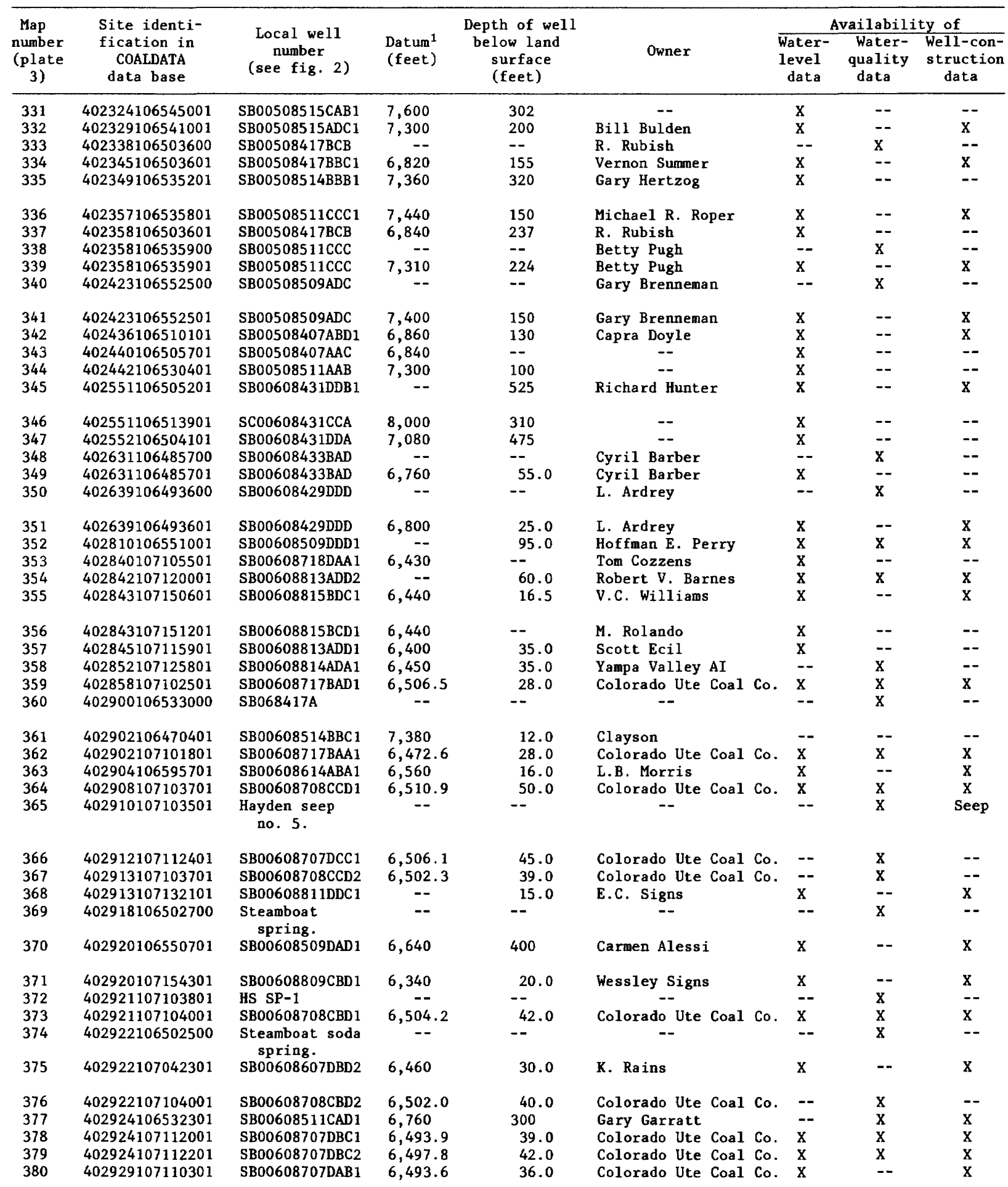


Table 5.--Ground-water site descriptions and availability of data collected by the U.S. Geological Survey--Continued

\begin{tabular}{|c|c|c|c|c|c|c|c|c|}
\hline \multirow{2}{*}{$\begin{array}{l}\text { Map } \\
\text { number } \\
\text { (plate } \\
3 \text { ) }\end{array}$} & \multirow{2}{*}{$\begin{array}{l}\text { Site identi- } \\
\text { fication in } \\
\text { COALDATA } \\
\text { data base }\end{array}$} & \multirow[b]{2}{*}{$\begin{array}{c}\text { Local well } \\
\text { number } \\
\text { (see fig. 2) }\end{array}$} & \multirow[b]{2}{*}{$\begin{array}{l}\text { Datum }{ }^{1} \\
\text { (feet) }\end{array}$} & \multirow{2}{*}{$\begin{array}{l}\text { Depth of well } \\
\text { below land } \\
\text { surface } \\
\text { (feet) }\end{array}$} & \multirow[b]{2}{*}{ Owner } & \multicolumn{3}{|c|}{ Availability of } \\
\hline & & & & & & $\begin{array}{l}\text { Water- } \\
\text { level } \\
\text { data }\end{array}$ & $\begin{array}{l}\text { Water- } \\
\text { quality } \\
\text { data }\end{array}$ & $\begin{array}{l}\text { Well-con- } \\
\text { struction } \\
\text { data }\end{array}$ \\
\hline 381 & 402930107105101 & SB00608707DAA1 & $6,495.1$ & 37.0 & Colorado Ute Coal Co. & $\mathrm{X}$ & $\mathrm{X}$ & $\mathrm{X}$ \\
\hline 382 & 402930107105201 & SB00608707DAA2 & $6,495.3$ & 38.0 & Colorado Ute Coal Co. & $x$ & $\mathrm{X}$ & $\mathrm{X}$ \\
\hline 383 & 402931106565501 & SB00608508CAB1 & -- & 12.0 & Pat Tellier & -- & -- & $\mathrm{X}$ \\
\hline 384 & 402932107110101 & SB00608707ADC1 & $6,493.6$ & 41.0 & Colorado Ute Coal Co. & $\mathrm{X}$ & $\mathrm{X}$ & $\mathrm{x}$ \\
\hline 385 & 402932107112001 & SB00608707DBB 1 & $6,499.6$ & 49.0 & Colorado Ute Coal Co. & $x$ & $\mathrm{X}$ & $\mathbf{x}$ \\
\hline 386 & 402933107110201 & SB00608707ADC2 & $6,490.8$ & 35.5 & Colorado Ute Coal Co. & $\mathbf{X}$ & $\mathbf{X}$ & $\mathrm{X}$ \\
\hline 387 & 402933107110701 & SB00608707ADC3 & $6,490.4$ & 36.0 & Farrington Carpenter & $\mathrm{X}$ & $\mathrm{X}$ & $\mathrm{X}$ \\
\hline 388 & 402933107110702 & SB00608707ADC4 & $6,485.6$ & 29.0 & Colorado Ute Coal Co. & $\mathbf{x}$ & $\mathrm{X}$ & $\mathrm{x}$ \\
\hline 389 & 402933107111301 & SB00608707ACD1 & $6,497.8$ & 42.0 & Colorado Ute Coal Co. & $\mathrm{X}$ & $X$ & $\mathbf{x}$ \\
\hline 390 & 402933107112201 & SB00608707ACC1 & $6,496.3$ & 43.0 & Colorado Ute Coal Co. & $\mathrm{x}$ & $\mathrm{X}$ & $\mathrm{x}$ \\
\hline 391 & 402934107111301 & SB00608707ACD2 & $6,496.7$ & 43.0 & Farrington Carpenter & $\mathrm{X}$ & $\mathrm{X}$ & $\mathrm{X}$ \\
\hline 392 & 402935107105301 & SB00608707ADD 1 & $6,493.1$ & 38.0 & Farrington Carpenter & $\mathrm{X}$ & $\mathrm{X}$ & $\mathrm{X}$ \\
\hline 393 & 402937106503100 & $\begin{array}{l}\text { Steamboat sulfur } \\
\text { spring. }\end{array}$ & -- & -- & $=-$ & -- & $\mathrm{X}$ & -- \\
\hline 394 & 402937107111401 & SB00608707ACD3 & $6,487.1$ & 35.0 & Farrington Carpenter & $\mathrm{X}$ & $\mathrm{X}$ & $x$ \\
\hline 395 & 402941106523101 & SB00608512BCA1 & 6,760 & 265 & Peter Stanko & $\mathrm{X}$ & -- & - \\
\hline 396 & 402949107135201 & SB00608811BAC1 & 6,400 & 20.0 & James Camilletti & $\mathrm{X}$ & -- & -- \\
\hline 397 & 402954107103501 & SB00608708BBA1 & 6,440 & -- & Carpenter & $\mathrm{X}$ & -- & -- \\
\hline 398 & 402955107123501 & SB00608812BAA1 & -- & -- & - $\quad-$ & $\mathrm{X}$ & -- & -- \\
\hline 399 & 402957107103301 & SB00608708BAB 1 & -- & 10.6 & -- & $\mathrm{X}$ & $\mathrm{X}$ & -- \\
\hline 400 & 402959107131101 & SB00608802DDD1 & 6,400 & -- & -- & $\mathrm{X}$ & -- & -- \\
\hline 401 & 402620107072960 & Pond 004 & 7,220 & -- & -- & -- & $\mathbf{X}$ & -- \\
\hline 402 & 402437107051704 & SBU287 & $7,029.97$ & 175 & -- & -- & $\mathrm{X}$ & -- \\
\hline 403 & 402437107051703 & SBW287 & $7,031.97$ & 164 & -- & -- & $\mathrm{X}$ & -- \\
\hline 404 & 402437107051701 & SBL287 & $7,031.96$ & 98.5 & -- & -- & $\mathrm{X}$ & -- \\
\hline 405 & 402359107054404 & Scu287 & $6,957.14$ & 122 & -- & -- & $\mathrm{X}$ & -- \\
\hline 406 & 402359107054402 & SCI287 & $6,957.08$ & 70.0 & -- & -- & $\mathrm{X}$ & -- \\
\hline 407 & 402359107054401 & SCL287 & $6,957.12$ & 30.0 & -- & -- & $\mathrm{x}$ & -- \\
\hline 408 & 402359107054403 & SCW287 & $6,957.05$ & 90.0 & -- & -- & $\mathrm{X}$ & -- \\
\hline 409 & 402358107054501 & $\operatorname{scss} 87$ & -- & -- & -- & -- & $\mathrm{X}$ & -- \\
\hline 410 & 402401107054701 & $\operatorname{scs} 1487-62$ & $6,975.51$ & 51.5 & -- & -- & $\mathrm{X}$ & -- \\
\hline 411 & 402401107054702 & $\operatorname{scs} 2487-63$ & $6,979.68$ & 37.5 & -- & -- & $\mathrm{X}$ & -- \\
\hline 412 & 402401107054703 & $\operatorname{scs} 3487-64$ & $6,982.73$ & 37.2 & -- & -- & $\mathrm{x}$ & -- \\
\hline 413 & 402401107054704 & $\operatorname{scs} 4487-65$ & $6,997.08$ & 25.0 & -- & -- & $\mathrm{X}$ & - \\
\hline 414 & 402454107071605 & SSU187 & $6,860.73$ & 127 & -- & -- & $\mathrm{X}$ & -- \\
\hline 415 & 402454107071604 & SSU487 & $6,860.73$ & 105 & -- & -- & $\mathrm{X}$ & -- \\
\hline 416 & 402454107071602 & SSI287 & $6,859.84$ & 79.8 & -- & -- & $\mathrm{X}$ & -- \\
\hline 417 & 402454107071603 & SSW287 & $6,859.91$ & 98.5 & -- & -- & $\mathrm{X}$ & -- \\
\hline 418 & 402454107071601 & SSL287 & $6,860.64$ & 58.0 & -- & -- & $\mathrm{X}$ & -- \\
\hline 419 & 402454107071606 & SSD487 & $6,859.74$ & 195 & -- & -- & $\mathrm{X}$ & -- \\
\hline 420 & 402454107071201 & SSSS87 & -- & -- & -- & - & $x$ & -- \\
\hline 421 & 402456107071101 & $\operatorname{sSS} 1487-59$ & $6,898.61$ & 33.0 & -- & -- & $\mathrm{X}$ & -- \\
\hline 422 & 402456107071102 & SSS $2487-60$ & $6,892.55$ & 22.8 & -- & -- & $\mathrm{x}$ & -- \\
\hline 423 & 402456107071103 & SSS3487-61 & $6,891.86$ & 26.0 & -- & -- & $\mathrm{X}$ & -- \\
\hline 424 & 402455107073001 & SZL287 & $6,822.14$ & 181 & -- & -- & $\mathrm{X}$ & -- \\
\hline 425 & 402455107073002 & SZI287 & $6,818.60$ & 222 & -- & -- & $\mathrm{X}$ & -- \\
\hline 426 & 402455107073004 & SZU287 & $6,820.28$ & 265 & -- & -- & $\mathrm{X}$ & -- \\
\hline 427 & 402455107073003 & SZW487 & $6,817.03$ & 237 & -- & -- & $\mathrm{X}$ & -- \\
\hline 428 & 401114107493601 & SB00309333ACD1 & -- & -- & -- & -- & $\mathrm{x}$ & -- \\
\hline 429 & 401142107514801 & SB00309330DCA & 7,520 & 394 & D. Smith -M. Crawford & - & -- & $\mathrm{X}$ \\
\hline 430 & 401207107505901 & SB00309329BDD & 7,400 & 275 & David Smith & $\mathrm{x}$ & -- & $x$ \\
\hline 431 & 401225107493301 & SB00309328ABA & -- & 560 & George Sturgeon & $\mathrm{X}$ & $\mathrm{x}$ & $\mathrm{X}$ \\
\hline 432 & 401332107481501 & SB00309315DDB & 6,770 & 259 & Harry Durham, Jr. & $\mathrm{X}$ & $\mathrm{X}$ & $\mathbf{X}$ \\
\hline 433 & 401351107362901 & SB00309116DAA & 7,090 & -- & Larry Osborn & $\mathrm{X}$ & -- & -- \\
\hline 434 & 401402107524501 & SB00309413AAC1 & 7,300 & 100 & Texaco Oil Co. & -- & $\mathrm{x}$ & $\mathrm{X}$ \\
\hline 435 & 401407107352301 & SB00309115AAC & -- & 8.0 & Leonard Forbes & -- & -- & -- \\
\hline
\end{tabular}


Table 5.--Ground-water site descriptions and availability of data collected by the U.S. Geological Survey--Continued

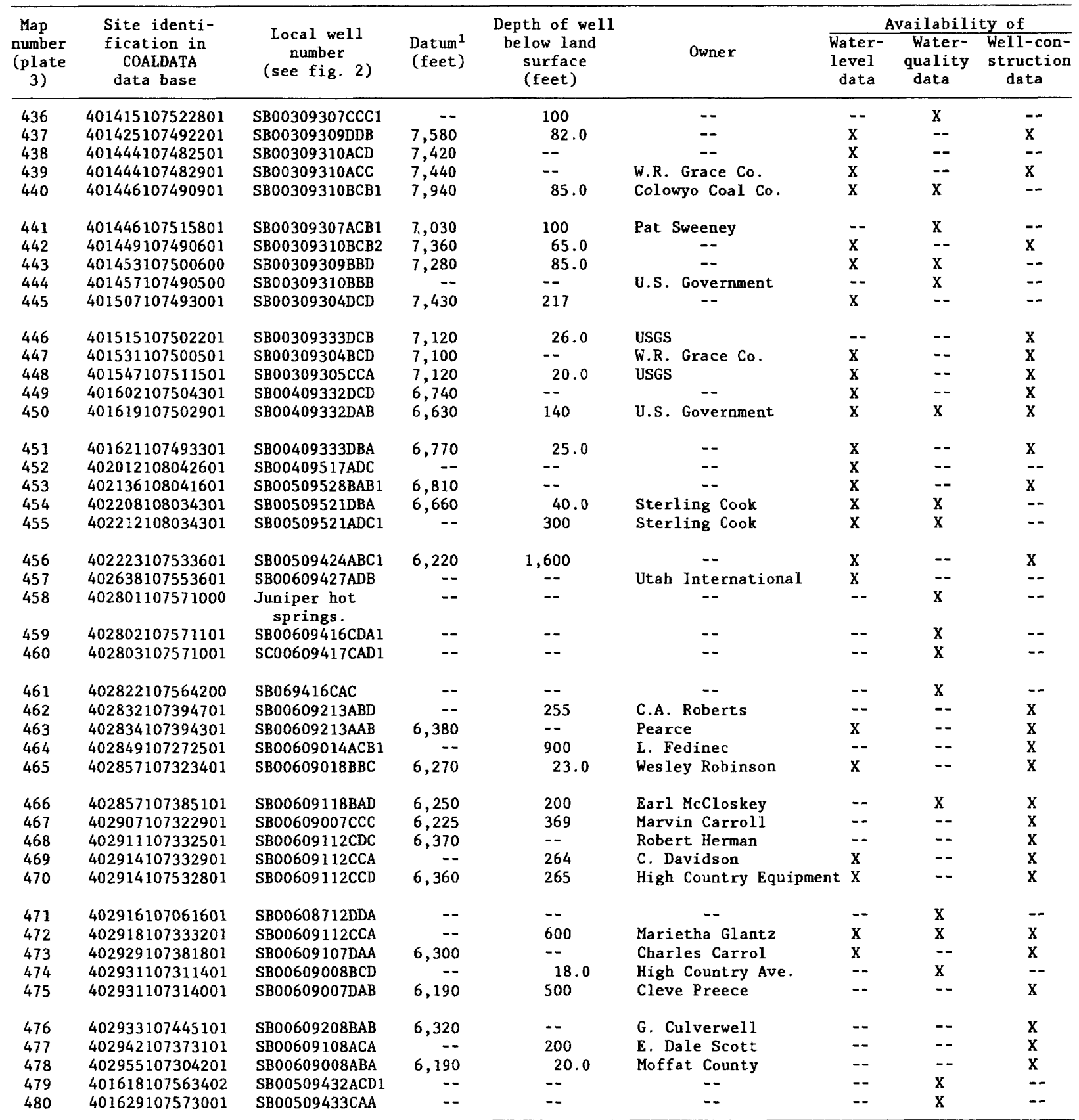

${ }^{1}$ Number of significant figures varies because some of the wells are listed as surveyed; however, these results have not been confirmed. 
Surface-water sites are listed in tables 2 and 3 . The locations of these sites are shown on plate 1 . Sites that have discharge and water-quality data collected by agencies other than the U.S. Geological Survey are listed in table 2. Sites that have discharge and water quality data collected by the U.S. Geological Survey are listed in table 3. The sites are first identified by site identification. The site identification is a unique number that identifies each site and is the number used to retrieve data for a site. The eight-digit site-identification number is a unique number assigned to a surface-water site that is operated by the U.S. Geological Survey. The 15-digit site-identification number is composed of a 6-digit latitude, a 7-digit longitude, and a 2-digit sequence number. The station name describes the location of the site using names of streams, towns, roads, and so forth. In table 2, the mine and the mine's site ID are used to correlate the COALDATA site identification (site identification, station name, township, range, section) with local non-standard identification (mine's site ID). The mines are identified using three-letter codes, which are defined in the table. The datum is the altitude above sea level (see explanation of sea level following the conversion factor table) of the reference point used at a site for measuring the height of the water surface. All the sites in table 3 are U.S. Geological Survey surface-water sites in the study area that have data in the U.S. Geological Survey data base. The site type column in tables 2 and 3 describes a site as having a continuous recording gage (GS) or chemical sampling site only (C). The last two columns in tables 2 and 3 indicate what kind of hydrologic data is available for a site.

Ground-water site descriptions are listed in tables 4 and 5 . Groundwater sites used by agencies other than the U.S. Geological Survey that have data in the COALDATA data base are listed in table 4. Ground-water sites used by the U.S. Geological Survey that have data in the U.S. Geological Survey data base are listed in table 5. The general locations of these ground-water sites are shown on plates 2 and 3 . The unique 15-digit site identification for a ground-water site listed in tables 4 and 5 is established the same way as with a surface-water site. The local well number in tables 4 and 5 is used to locate the ground-water site (well or spring) easily using the township, range, section, and subdivisions of the section (fig. 2). The mine and site ID of the mine in table 4 is the same as in table 2. The datum in tables 4 and 5 is the altitude of the land surface in feet above sea level. Ground-water levels are commonly measured in feet below land surface. In table 5, the owners of ground-water sites (wells or springs) sampled by the U.S. Geological Survey include private individuals, companies, and the U.S. Government. The types of data available are listed in the last three columns of tables 4 and 5 . Locations of the ground-water sites are shown in the maps on plates 2 and 3. 
Surface-water data include discharge measurements. Surface-water station descriptions include the following: Latitude and longitude; township, range, and section; a narrative description of the location of the gage, measurement site, or sampling site; codes for county and state; station type (gaging station, flume); mine name; and mine's site identification. An example of a computer printout of surface-water data is listed in table 6 in the "Supplemental Data" section at the back of the report.

Ground-water data in the system consist of site descriptions and water levels. Water levels are reported in feet below land surface. Site descriptions, which are the major components of ground-water data in COALDATA, include: Latitude and longitude; township, range, and section; state, and county codes; station type; data reliability, type of ground-water site; primary use of site; and date of water level, if a measurement was taken. Other data that may be available in the ground-water site description include: Depths to geologic formations; well-construction information; and hydrologic information about the aquifer. An example of a computer printout of groundwater data is listed in table 7 in the "Supplemental Data" section at the back of the report.

Quality of water data in the system include chemical analyses of water samples of surface water and ground water. There is an extensive list of chemical, physical, and biological constituents that can be stored in the quality of water data base. The constituents stored in the COALDATA data base are listed in table 1. An example of a computer printout of quality of water data is listed in table 8 in the "Supplemental Data" section at the back of the report.

Several sites are listed in tables 2 and 4 that have only a site location. These sites were included in the tables because, even though there are no data for them in the COALDATA data base, the sites are part of the hydrologic data-collection network established by the indicated coal mine and there may be data available elsewhere. 
SUPPLEMENTAL DATA 


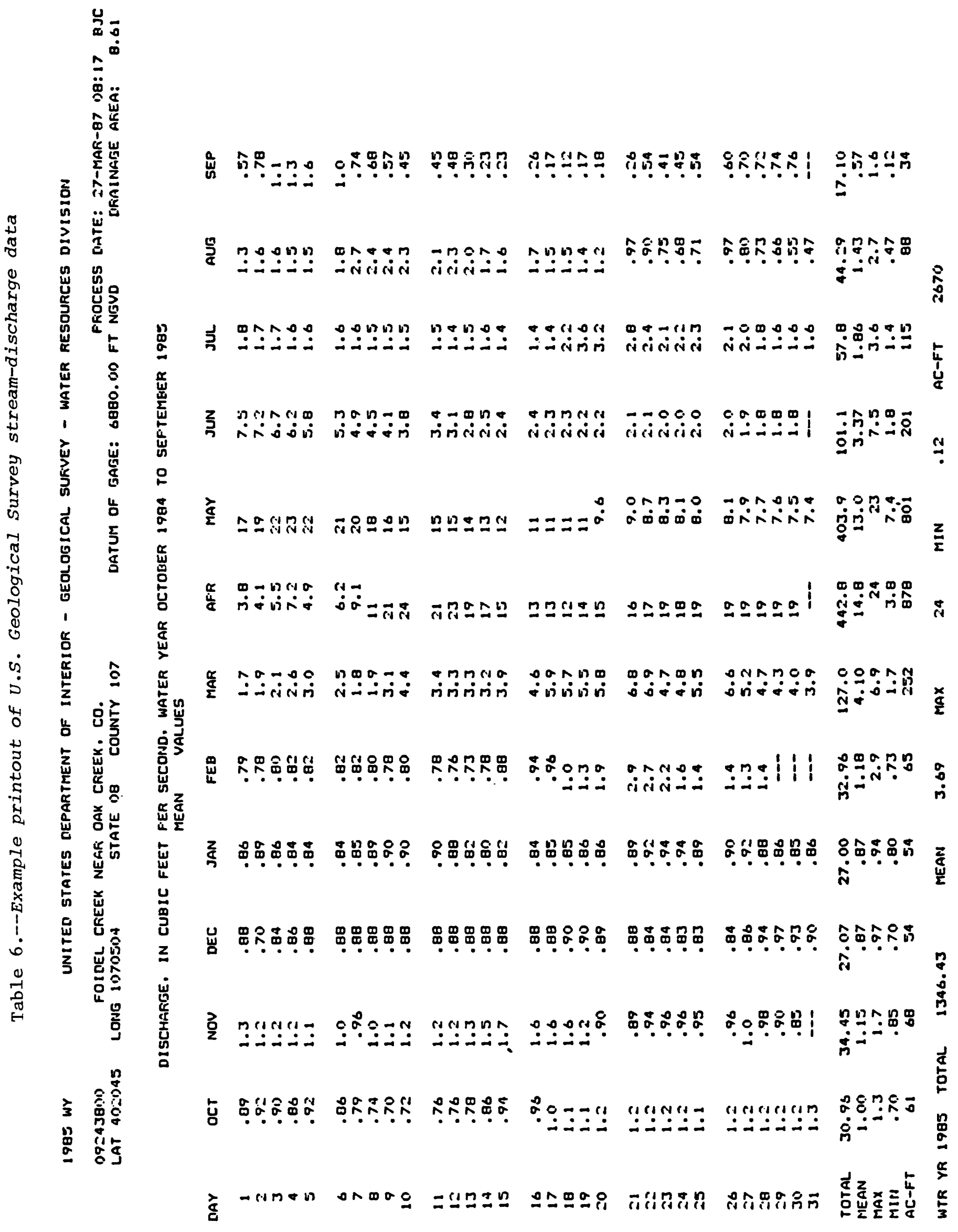




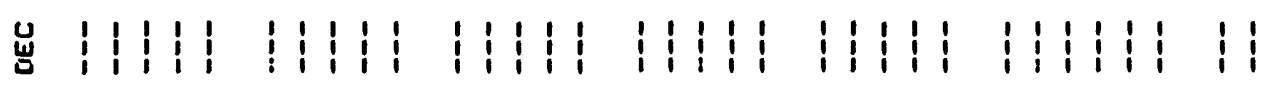

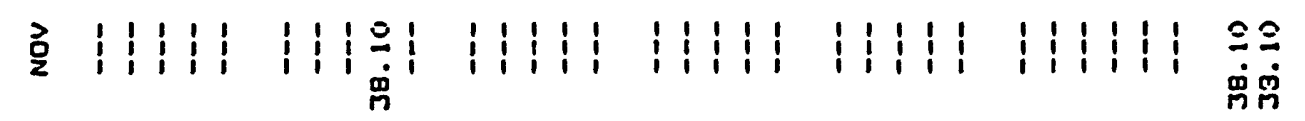

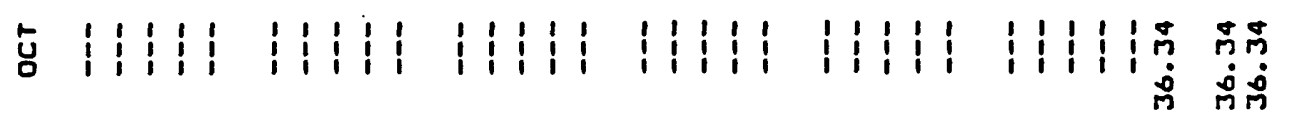

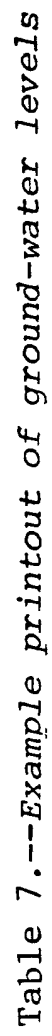

\&

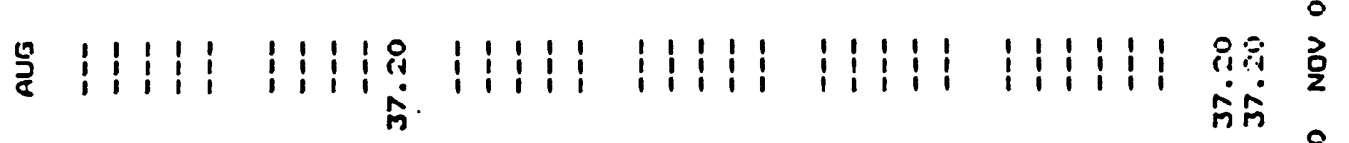

声

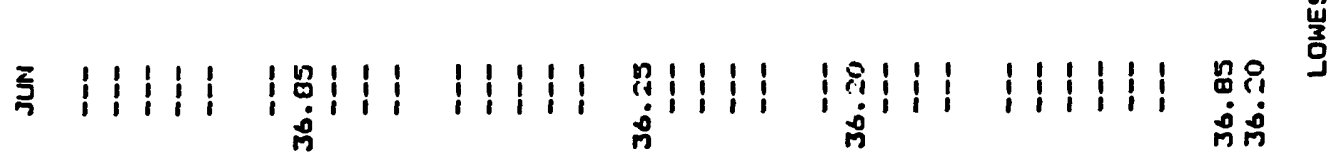

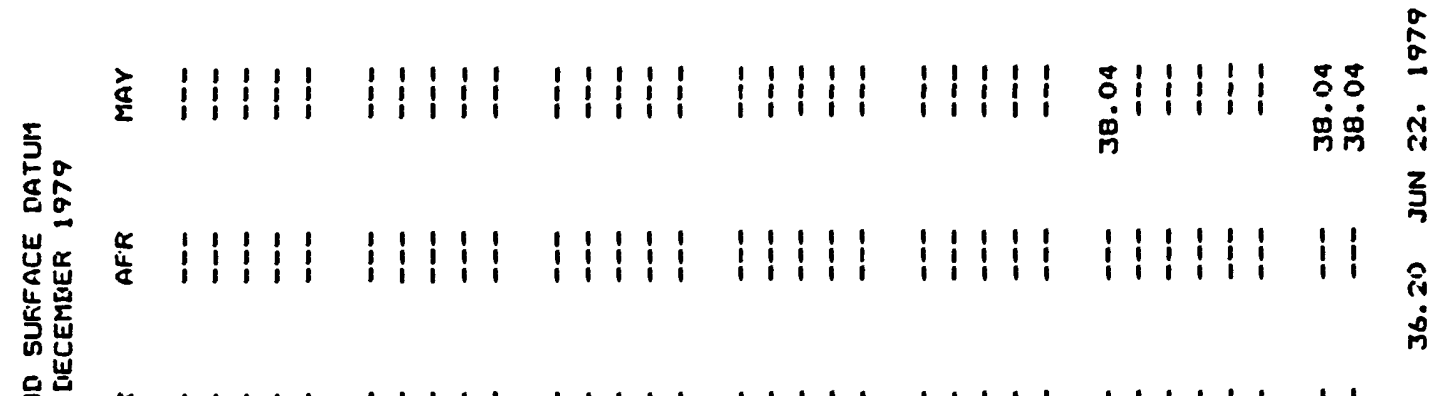

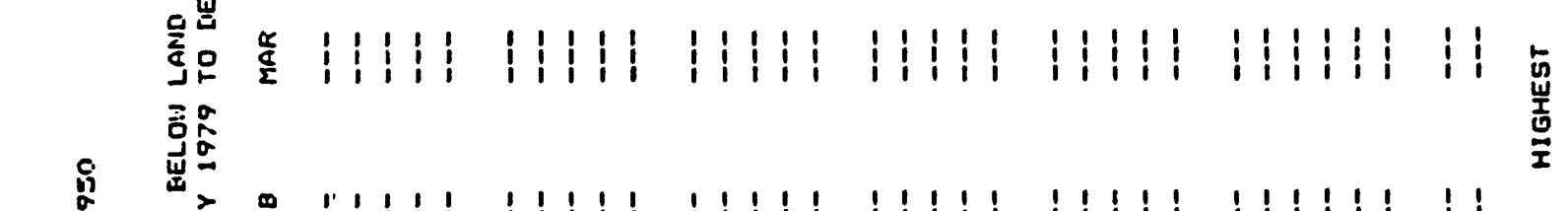

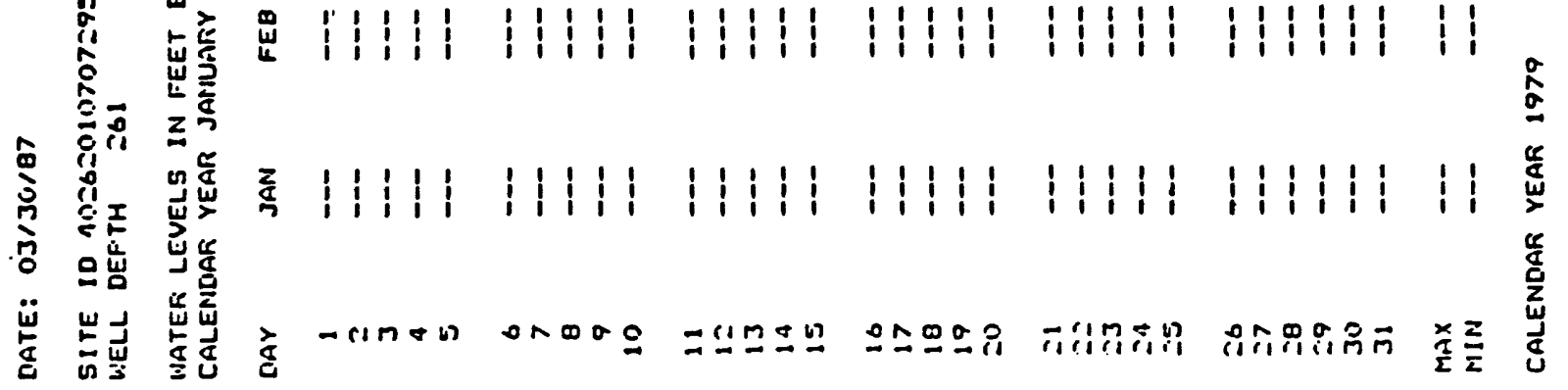


â

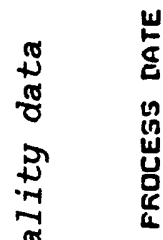

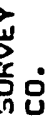

ब्र

突峲

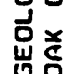

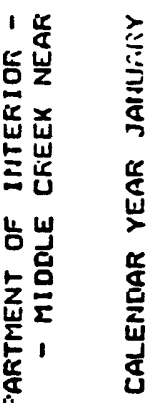

崖

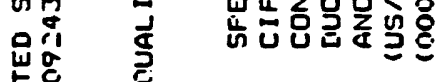

$\bar{z}$

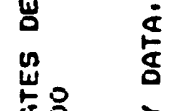

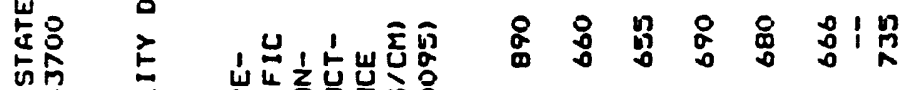

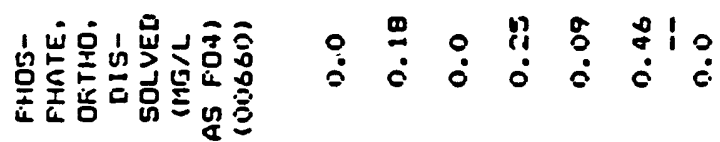

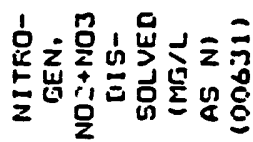

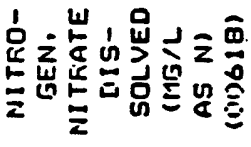

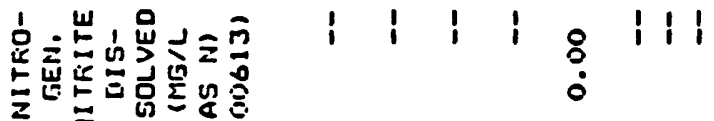

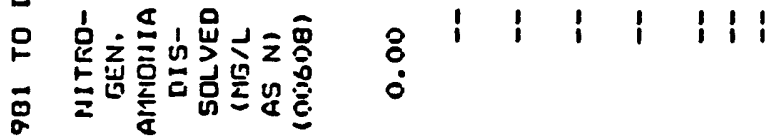

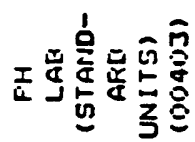

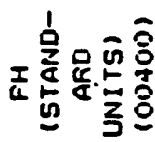

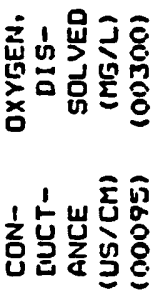

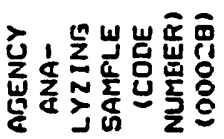

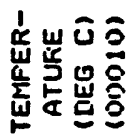

岁

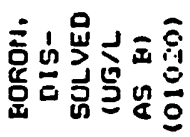

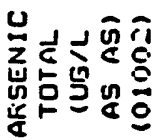

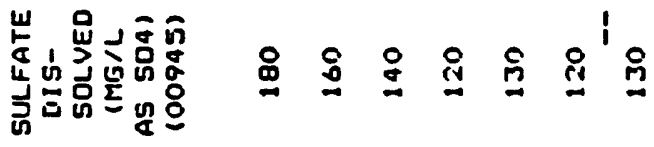

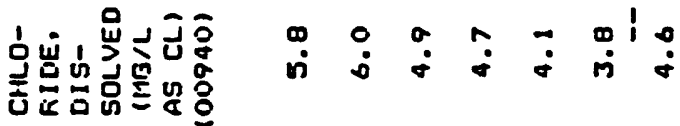

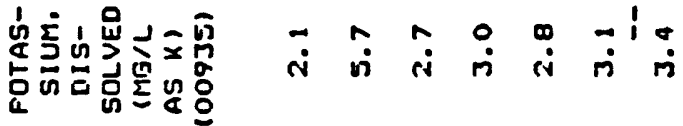

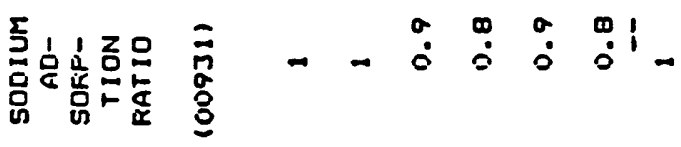

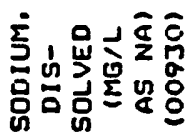

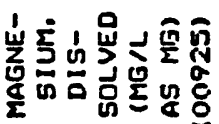

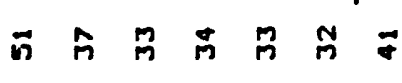

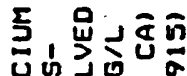

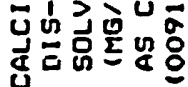
ก量

\&

岂

壷

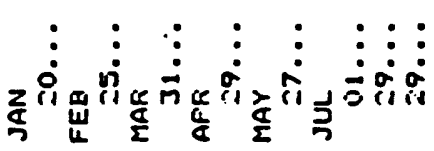

$\frac{\omega}{\underline{\Xi}}$

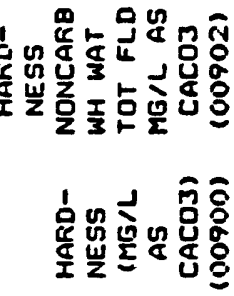

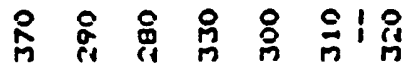

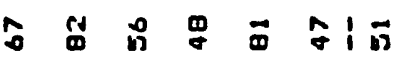

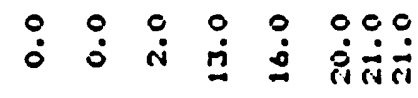

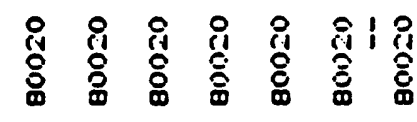

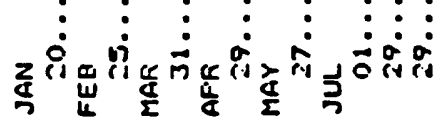

